\title{
Asymptotic profiles for the second grade fluids equations in $\mathbb{R}^{3}$
}

\author{
Olivier Coulaud
}

Communicated by Y. Charles Li, received February 28, 2014.

\begin{abstract}
In the present paper, we study the long time behaviour of the solutions of the second grade fluids equations in $\mathbb{R}^{3}$. Using scaling variables and energy estimates in weighted Sobolev spaces, we describe the first order asymptotic profiles of these solutions. In particular, we show that the solutions of the second grade fluids equations converge to self-similar solutions of the heat equation, which are explicit and depend on the initial data. Since this phenomenon occurs also for the Navier-Stokes equations, it shows that the fluids of second grade behave asymptotically like Newtonian fluids.
\end{abstract}

\section{Contents}

1. Introduction

2. First order asymptotics and preliminary results

3. Approximate solutions

4. Energy estimates

5. Proof of Theorem 2.2

\section{Introduction}

Since one can find a lot of non-Newtonian fluids in the nature or in the industry, their mathematical study is a significant topic of research. In this paper, we investigate the long time behaviour of a particular class of non-Newtonian fluids, namely the second grade fluids. The equations which describe such fluids have been introduced from a mathematical point of view in 1974 by Dunn and Fosdick in [10] and have been the topic of many research works in mathematics. These fluids are a particular case of a large class of non-Newtonian fluids, called fluids of differential

1991 Mathematics Subject Classification. 35, 76. solutions.

Key words and phrases. Second grade fluids equations, asymptotic profiles, self-similar 
type, or Rivlin-Ericksen fluids (see [28]). The constitutive laws of the differential fluids are given through the Rivlin-Ericksen tensors, defined recursively by

$$
\begin{aligned}
& A_{1}=\nabla u+(\nabla u)^{t}, \\
& A_{k}=\partial_{t} A_{k-1}+u . \nabla A_{k-1}+(\nabla u)^{t} A_{k-1}+A_{k-1} \nabla u,
\end{aligned}
$$

where $u: \mathbb{R}^{d} \rightarrow \mathbb{R}^{d}$ is a vector field which represents the velocity of a fluid filling a domain of $\mathbb{R}^{d}, d=2,3$. According to this model, the equations of the fluids of grade $n \in \mathbb{N}$ are obtained by considering the stress tensor

$$
\sigma=-p I+Q\left(A_{1}, A_{2}, \ldots, A_{n}\right),
$$

where $p$ is the pressure of the fluid and $Q$ is a polynomial function of degree $n$. Notice that the fluids of grade 1 correspond to the Newtonian fluids, whose velocity fields are the solutions of the Navier-Stokes equations. According to the model of Dunn and Fosdick (see [10]), the constitutive law of the second grade fluids is obtained via the stress tensor

$$
\sigma=-p I+\nu A_{1}+\alpha_{1} A_{2}+\alpha_{2} A_{1}^{2},
$$

where $\nu>0$ is the dynamic viscosity of the fluid, $\alpha_{1}>0$ and $\alpha_{2} \in \mathbb{R}$. In [10], thermodynamic considerations led the authors to assume that $\alpha_{2}=-\alpha_{1}$. Consequently, we replace $\alpha_{1}$ by $\alpha$. Introduced in the equations of conservation of momentum and assuming that the density of the fluid is constant and equals 1, the tensor $\sigma$ leads to the system of equations

$$
\begin{aligned}
& \partial_{t}(u-\alpha \Delta u)-\nu \Delta u+\operatorname{curl}(u-\alpha \Delta u) \wedge u+\nabla p=0, \\
& \operatorname{div} u=0, \\
& u_{\mid t=0}=u_{0},
\end{aligned}
$$

where $\wedge$ denotes the classical vectorial product on $\mathbb{R}^{3}, p$ is the pressure and $u_{0}$ is the initial data. In the two-dimensional case, we have used the convention that $u=\left(u_{1}, u_{2}, 0\right)$ and $\operatorname{curl} u=\left(0,0, \partial_{1} u_{2}-\partial_{2} u_{1}\right)$.

Several existence and uniqueness results have been obtained for this system of equations, mainly on a bounded domain $\Omega$ of $\mathbb{R}^{2}$ or $\mathbb{R}^{3}$ with Dirichlet or periodic boundary conditions (see for instance $[\mathbf{1}],[\mathbf{2}],[\mathbf{6}],[\mathbf{7}],[\mathbf{8}],[\mathbf{9}],[\mathbf{1 5}],[\mathbf{2 6}]$ or $[\mathbf{2 5}]$ ). The first existence and uniqueness result has been obtained by Cioranescu and El Hacène in 1984 in [6]. They have shown, on a bounded set of $\mathbb{R}^{d}, d=2,3$, with homogeneous boundary conditions, that there exists a unique weak solution to (1.1) belonging to the space $L^{\infty}\left([0, T], H^{3}(\Omega)^{d}\right)$, where $T>0$ and $H^{s}(\Omega)$ denotes the Sobolev space of order $s$. Besides, this solution is global in time when the space dimension is 2. This result is based on a priori estimates and a Galerkin approximation with a basis of eigenfunctions corresponding to the scalar product associated to the operator curl $(u-\alpha \Delta u)$. In the same case, using the Schauder fixed point Theorem, Galdi, Grobbelaar-Van Dalsen and Sauer established the existence and uniqueness of classical solutions to (1.1) when the data belong to $H^{m}(\Omega)$, with $m \geq 5$ (see $[\mathbf{1 4}]$ ). They also have shown that these solutions are global in time, provided that the initial data are small enough in $H^{m}(\Omega)$. Later, Cioranescu and Girault improved the results of $[\mathbf{6}]$ and $[\mathbf{1 4}]$ and showed that the local weak solutions belonging to $H^{3}(\Omega)$ are actually global in time in dimension 3 if the data are small enough and are strong solutions if the data belong to $H^{m}, m \geq 4$ (see [5]). Finally, Bresch and Lemoine have generalized the results of $[\mathbf{1 4}],[\mathbf{6}]$ and $[\mathbf{5}]$ in dimension 3 in establishing the existence and uniqueness of local solutions belonging to the 
space $W^{2, r}(\Omega)$ with $r>3$. Furthermore, they have shown that these solutions are global in time if the initial data are small enough in $W^{2, r}(\Omega)$ (see $[\mathbf{2}]$ ). In this work, instead of applying a Galerkin approximation, the authors used Schauder's fixed point Theorem.

In the present paper, we are interested in the description of the asymptotic profiles of the solutions of second grade fluids equations. In what follows, we consider a second grade fluid which fills the whole space $\mathbb{R}^{3}$, without any forcing term applied to it. In this case, if the initial data are small enough, the solutions of such a system tend to 0 when the time $t$ goes to infinity. The aim of this study is to investigate the way that these solutions go to 0 . More precisely, we will show that the solutions of (1.1) behave asymptotically like self-similar solutions to the heat equation, which are smooth and that we can compute explicitly from the data. In this article, we restrict ourselves to the study of the first order asymptotic profile, that is to say that the speed of the convergence of the solutions of (1.1) to explicit smooth functions is limited by spectral considerations, which are explained below. For the Navier-Stokes equations, there already exist several results that describe the asymptotic profiles of the solutions. In dimension 2 and 3, Gallay and Wayne have shown in $[\mathbf{1 6}]$ and $[\mathbf{1 7}]$ that the first order asymptotic profiles of the solutions of the Navier-Stokes equations are given up to a constant by smooth Gaussian functions which are self-similar solutions to the heat equation. These results hold with restrictions on the size of the data, but, in dimension 2 , the convergence has been generalized to the case of any data in $[\mathbf{1 8}]$. For this work, the authors applied arguments that come from the study of dynamical systems. In fact, they have shown the existence of a finite-dimensional manifold locally invariant by the semiflow associated to the Navier-Stokes equations. Then, they proved that the solutions of the Navier-Stokes equations are locally attracted by this manifold, and consequently behave like the solutions on it. The study of the dynamics of the Navier-Stokes equations onto this manifold gave them the description of the first and second order asymptotic profiles. The asymptotic profiles of the solutions of the equations of second grade fluids have been studied in $\mathbb{R}^{2}$ by Jaffal-Mourtada (see $[\mathbf{2 3}]$ ). She has shown, under smallness assumptions on the data, that the first order asymptotic profiles of the solutions of the second grade fluids equations are the same as the ones described by Gallay and Wayne in [16] for the Navier-Stokes equations. However, the method that she used in [23] is slightly different from the one used in [16]. Indeed, instead of showing the existence of an invariant manifold, the author performed energy estimates in various functions spaces, notably weighted Sobolev spaces. The concrete interpretation of this result is that, in dimension 2 , the fluids of second grade behave asymptotically like Newtonian fluids, at the first order. In this article, we are interested in the generalization of this result to the dimension 3. Notice that there are significant differences in the asymptotic behaviour of the solutions of the Navier-Stokes equations between the cases of $\mathbb{R}^{2}$ and $\mathbb{R}^{3}$. Indeed, in dimension 2, the asymptotic profiles of the Navier-Stokes equations are given up to a constant by a Gaussian function called the Oseen vortex sheet. In dimension 3 , the first order asymptotic profiles of the solutions are defined as the linear combination of three distinct smooth functions (see Section 2).

Actually, the system that we study in this article is not exactly (1.1) but the one 
satisfied by the vorticity $w=\operatorname{curl} u=\partial_{1} u_{2}-\partial_{2} u_{1}$. The motivation to do this comes from the fact that, due to spectral considerations which will be explained more precisely later, we have to solve the second grade fluids equations in weighted Lebesgue spaces. Unfortunately, the equations of motion (1.1) do not preserve in general those functions spaces, on the contrary to the vorticity equations. We assume, for the sake of simplicity, that $\nu=1$ and consider initial vorticity data $w_{0}$. Taking formally the curl of (1.1), we get the vorticity system of equations

$$
\begin{aligned}
& \partial_{t}(w-\alpha \Delta w)-\Delta w+\operatorname{curl}((w-\alpha \Delta w) \wedge u)=0, \\
& \operatorname{div} u=\operatorname{div} w=0, \\
& w_{\mid t=0}=w_{0} .
\end{aligned}
$$

This system is actually autonomous. Indeed, provided that $w$ is sufficiently smooth, the divergence free vector field $u$ can be recovered from $w$ via the Biot-Savart law, which is a way to get a divergence free vector field from its given vorticity. It is defined by the formula

$$
u(x)=-\frac{1}{4 \pi} \int_{\mathbb{R}^{3}} \frac{(x-y) \wedge w(y)}{|x-y|^{3}} d y .
$$

In Section 2, more details are given on the Biot-Savart law and its properties (see Lemma 2.4). In this article, we show that the solutions of the system (1.2) behave asymptotically like vector fields whose components are self-similar solutions to the well known heat equation, that is to say under the form

$$
(t, x) \rightarrow \frac{1}{(t+T)^{2}} F\left(\frac{x}{\sqrt{t+T}}\right),
$$

where $F$ is a vector field of $\mathbb{R}^{3}$ and $T$ is a positive constant.

We introduce now an useful tool in the study of the asymptotics of solutions to partial differential equations, that is scaled variables or self-similar variables. In order to define those variables, we set a positive constant $T$, and we will always assume $T \geq 1$. This constant is introduced in order to establish the convergence of the solutions to their asymptotic profiles without any restriction on the size of the constant $\alpha$. As it is explained below, the constant $T$ will be chosen large enough to have $\frac{\alpha}{T}$ small enough. For a solution $w$ of the system (1.2), we define $W$ and $U$ through the change of variable $X=\frac{x}{\sqrt{t+T}}$ and $\tau=\log (t+T)$. More precisely, we set

$$
\begin{aligned}
& w(t, x)=\frac{1}{t+T} W\left(\log (t+T), \frac{x}{\sqrt{t+T}}\right), \\
& u(t, x)=\frac{1}{\sqrt{t+T}} U\left(\log (t+T), \frac{x}{\sqrt{t+T}}\right) .
\end{aligned}
$$

Equivalently, we have the equalities

$$
\begin{aligned}
& W(\tau, X)=e^{\tau} w\left(e^{\tau}-T, e^{\tau / 2} X\right), \\
& U(\tau, X)=e^{\tau / 2} u\left(e^{\tau}-T, e^{\tau / 2} X\right) .
\end{aligned}
$$

Actually, this change of variables is the one that correspond to the self-similar solutions of the heat equation in dimension 2. However, in the present paper, we are considering solutions which are defined on $\mathbb{R}^{3}$, but the final result that we obtain (see Corollary 2.1 below) do not depend on the change of variables that 
we consider. The advantage of defining $W$ and $U$ by the equality (1.4) lies in the fact that the equations that we obtain for $W$ and $U$ are the same as the ones obtained by the same method in dimension 2 . These variables have been initially introduced to study the asymptotic behaviours of solutions of parabolic equations, in particular to show the convergence to self-similar solutions (see $[\mathbf{1 1}],[\mathbf{1 2}],[\mathbf{1 3}]$ or [24]). Actually, this tool is also efficient to study the long-time behaviour of a lot of various equations, not necessarily parabolic ones. For instance, Gallay and Raugel used them to describe the first and second order asymptotic profiles of the solutions to damped waved equations (see [20]) and to show the stability of hyperbolic fronts (see [21]). Self-similar variables have been also used to study the asymptotic profiles of the Navier-Stokes equations (see [16], [17], [18] and [19]) and the second grade fluids equations in $\mathbb{R}^{2}$ (see $[\mathbf{2 3}]$ ). Assuming that $w$ is a solution of (1.2), a short computation shows that $W$ is a solution of the system

$$
\begin{aligned}
& \partial_{\tau}\left(W-\alpha e^{-\tau} \Delta W\right)-\mathcal{L}(W)+\operatorname{curl}\left(\left(W-\alpha e^{-\tau} \Delta W\right) \wedge U\right) \\
& +\alpha e^{-\tau} \Delta W+\alpha e^{-\tau} \frac{X}{2} \cdot \nabla \Delta W=0, \\
& \operatorname{div} U=\operatorname{div} W=0 \\
& W_{\mid \tau=\log (T)}=W_{0},
\end{aligned}
$$

where $\mathcal{L}$ is the linear differential operator defined by

$$
\mathcal{L}(W)=\Delta W+W+\frac{X}{2} \cdot \nabla W .
$$

We first emphasize that the system (1.6) is now non-autonomous and initialised at $\tau=\log (T)$, that is the reason why we introduced the constant $T$. Indeed, in what follows, we will choose $T$ large enough so that the product $\alpha e^{-\tau}$ is small enough, for all $\tau \geq \log (T)$. By doing this, we do not have to consider restrictions on the size of the parameter $\alpha$. We also notice that, in the first equality, several terms tend formally to 0 when $\tau$ goes to infinity. Actually, the main theorem of this article shows that the solutions of (1.6) converge when $\tau$ goes to infinity to particular solutions to the equality

$$
\partial_{\tau} W_{\infty}=\mathcal{L}\left(W_{\infty}\right)
$$

More precisely, the aim of this article is to decompose $W$ on the spectrum of $\mathcal{L}$ on an appropriate space of functions and to show that the asymptotic behaviour of $W$ is dominated by his first order asymptotic profile, that is to say the projection of $W$ onto the eigenspace corresponding to the first eigenvalue of $\mathcal{L}$. Additionally, this projection satisfies the equality (1.7). Now, we introduce the weighted Lebesgue spaces, which are suitable for the study of the spectrum of $\mathcal{L}$. For every $m \in \mathbb{N}$, one defines $L^{2}(m)$, given by

$$
L^{2}(m)=\left\{u \in L^{2}\left(\mathbb{R}^{3}\right):\left(1+|x|^{2}\right)^{m / 2} u \in L^{2}\left(\mathbb{R}^{3}\right)\right\},
$$

where $|x|=\left(\sum_{i=1}^{3} x_{i}^{2}\right)^{1 / 2}$.

By the same way, for $m \in \mathbb{N}$ and $n \geq 2$, we define the weighted Sobolev spaces by

$$
\begin{aligned}
& H^{1}(m)=\left\{u \in L^{2}(m): \partial_{i} u \in L^{2}(m), i \in\{1,2,3\}\right\}, \\
& H^{n}(m)=\left\{u \in L^{2}(m): \partial_{i} u \in H^{n-1}(m), i \in\{1,2,3\}\right\} .
\end{aligned}
$$


The incompressibility condition on the vector fields $W$ and $U$ makes natural to consider the spaces

$$
\begin{aligned}
& \mathbb{L}^{2}(m)=\left\{u \in L^{2}(m)^{3}: \operatorname{div} u=0\right\}, \\
& \mathbb{H}^{2}(m)=\left\{u \in H^{2}(m)^{3}: \operatorname{div} u=0\right\},
\end{aligned}
$$

equipped with the norms

and

$$
\|u\|_{L^{2}(m)}=\left\|\left(1+|x|^{2}\right)^{\frac{m}{2}} u\right\|_{L^{2}},
$$

$$
\|u\|_{H^{2}(m)}=\left(\|u\|_{L^{2}(m)}^{2}+\|\nabla u\|_{L^{2}(m)}^{2}+\left\|\nabla^{2} u\right\|_{L^{2}(m)}^{2}\right)^{1 / 2}
$$

where we used the notations

$$
|\nabla u|=\left(\sum_{i, j=1}^{3}\left(\partial_{j} u_{i}\right)^{2}\right)^{1 / 2} \text { and }\left|\nabla^{2} u\right|=\left(\sum_{i, j, k=1}^{3}\left(\partial_{j} \partial_{k} u_{i}\right)^{2}\right)^{1 / 2}
$$

In [17], Gallay and Wayne show that the spectrum of $\mathcal{L}$ on $\mathbb{L}^{2}(m)$ is the union of the discrete spectrum

$$
\sigma_{d}(\mathcal{L})=\left\{-\frac{1}{2}(k+1), k \in \mathbb{N}\right\}
$$

and the continuous one

$$
\sigma_{c}(\mathcal{L})=\left\{\lambda \in \mathbb{C}: \operatorname{Re}(\lambda) \leq \frac{1}{4}-\frac{m}{2}\right\}
$$

In order to describe the first order asymptotic profiles of the solutions of (1.6), we need to have at least one isolated eigenvalue in the spectrum of $\mathcal{L}$. Looking at $\sigma_{c}(\mathcal{L})$, we notice that one can "push" the continuous spectrum to the left by choosing $m$ large enough. For this reason, we should work at least in the weighted space $\mathbb{L}^{2}(3)$, where -1 is an isolated eigenvalue of $\mathcal{L}$. In order to be closer to the optimal rate of convergence, we prefer working in $\mathbb{L}^{2}(4)$, where the discrete spectrum is $\sigma_{d}(\mathcal{L})=\left\{-1,-\frac{3}{2}\right\}$ and the continuous one is $\sigma_{c}(\mathcal{L})=\left\{\lambda \in \mathbb{C}: \operatorname{Re}(\lambda) \leq-\frac{7}{4}\right\}$. The main aim of this article is to show that one can decompose a solution $W$ of (1.6) into the form

$$
W(\tau)=\Omega(\tau)+R(\tau),
$$

where $\Omega$ is the projection of $W$ onto the eigenspace of $\mathcal{L}$ associated to the eigenvalue -1 and $R$ tends to 0 faster than $\Omega$ into $\mathbb{L}^{2}(4)$ when $\tau$ goes to infinity. In Section 2 , one establishes that

$$
\Omega(\tau)=\mathcal{O}\left(e^{-\tau}\right) \text { in } \mathbb{L}^{2}(4),
$$

and that the exists a positive constant $\theta, 1<\theta<\frac{3}{2}$ such that

$$
R(\tau)=\mathcal{O}\left(e^{-\theta \tau}\right) \text { in } \mathbb{L}^{2}(4) .
$$

Since the second isolated eigenvalue of $\mathcal{L}$ in $\mathbb{L}^{2}(4)$ is $\frac{3}{2}$, the optimal rate of convergence that one can obtain for such a first order description of the asymptotic profiles is $\theta=\frac{3}{2}$. Actually, the result that we obtain holds under smallness assumptions on the size of the data in $\mathbb{H}^{2}(4)$, and we will see in Section 2 that one can choose the rate of convergence as close as wanted to the optimal one, provided that the initial data are small enough. 


\section{First order asymptotics and preliminary results}

Before stating the main theorem of this paper, we have describe the eigenspace of $\mathcal{L}$ associated to the eigenvalue -1 . In $[\mathbf{1 7}$, appendix $\mathrm{A}]$, they show that the multiplicity of the eigenvalue -1 is 3 and that a suitable basis $\left\{f_{1}, f_{2}, f_{3}\right\}$ of the associated eigenspace $E_{-1}$ is given by

$$
f_{i}=\operatorname{curl}\left(G e_{i}\right), \quad i=1,2,3,
$$

where $G(X)=\frac{1}{(4 \pi)^{3 / 2}} e^{-\frac{|X|^{2}}{4}}$ and $\left\{e_{1}, e_{2}, e_{3}\right\}$ is the canonical basis of $\mathbb{R}^{3}$.

Through a short computation, we see that $f_{i}(X)=p_{i}(X) G(X), \quad i=1,2,3$, where

$$
p_{1}(X)=\frac{1}{2}\left(\begin{array}{c}
0 \\
-X_{3} \\
X_{2}
\end{array}\right), p_{2}(X)=\frac{1}{2}\left(\begin{array}{c}
X_{3} \\
0 \\
-X_{1}
\end{array}\right) \text { and } p_{3}(X)=\frac{1}{2}\left(\begin{array}{c}
-X_{2} \\
X_{1} \\
0
\end{array}\right) .
$$

In particular, the vector fields $p_{i}$ satisfy $\quad \operatorname{div} p_{i}=0 \quad$ and $\quad \operatorname{curl} p_{i}=e_{i}$. Integrating by parts, we also notice that

$$
\int_{\mathbb{R}^{3}} p_{i}(X) \cdot f_{j}(X) d X=\int_{\mathbb{R}^{3}} \operatorname{curl}\left(p_{i}(X)\right) \cdot\left(G(X) e_{j}\right) d X=\left(e_{i} \cdot e_{j}\right) \int_{\mathbb{R}^{3}} G(X) d X=\delta_{i j} .
$$

Furthermore, defining $\mathcal{L}^{*}=\Delta-\frac{X}{2} . \nabla-\frac{1}{2}$ the formal adjoint of $\mathcal{L}$, we check easily that

$$
\mathcal{L}^{*} p_{i}=-p_{i}
$$

With the basis $\left\{f_{1}, f_{2}, f_{3}\right\}$, the decomposition (1.8) can be written

$$
W(\tau)=\sum_{i=1}^{3} \beta_{i}(\tau) f_{i}+R(\tau)
$$

where $\beta_{i}(\tau) \in \mathbb{R}$.

As we can see in $[\mathbf{1 7}], \mathbb{L}^{2}(4)=E_{-1} \oplus \mathcal{W}$, where

$$
\mathcal{W}=\left\{f \in \mathbb{L}^{2}(4): \int_{\mathbb{R}^{3}} X_{i} f_{j}(X) d X=0, \quad i, j=1,2,3\right\} .
$$

Consequently, one has to choose $\beta_{i}$ such that $\int_{\mathbb{R}^{3}} X_{i} R_{j}(\tau, X) d X=0$, for $i, j \in$ $\{1,2,3\}$. To this end, we set

$$
\beta_{i}(\tau)=\int_{\mathbb{R}^{3}} p_{i}(X) \cdot W(\tau, X) d X
$$

In fact, assuming that $W \in \mathbb{L}^{2}(4)$ and using the divergence free property of $W$, it is easy to check that

$$
\begin{aligned}
\int_{\mathbb{R}^{3}} p_{1}(X) \cdot W(X) d X & =\int_{\mathbb{R}^{3}} X_{2} W_{3}(X) d X=-\int_{\mathbb{R}^{3}} X_{3} W_{2}(X) d X, \\
\int_{\mathbb{R}^{3}} p_{2}(X) \cdot W(X) d X & =\int_{\mathbb{R}^{3}} X_{3} W_{1}(X) d X=-\int_{\mathbb{R}^{3}} X_{1} W_{3}(X) d X, \\
\int_{\mathbb{R}^{3}} p_{3}(X) \cdot W(X) d X & =\int_{\mathbb{R}^{3}} X_{1} W_{2}(X) d X=-\int_{\mathbb{R}^{3}} X_{2} W_{1}(X) d X,
\end{aligned}
$$

and thus, using (2.2) and the decomposition (2.4), we can conclude that 


$$
\int_{\mathbb{R}^{3}} X_{i} R_{j}(X) d X=0, \text { for all } i, j \in\{1,2,3\} .
$$

The next lemma gives more details about $\beta_{i}$, and shows that the projection of $W$ onto $E_{-1}$ is actually a solution of (1.7).

Lemma 2.1. Let $W \in C^{o}\left(\left[\tau_{0}, T\right), \mathbb{H}^{2}(4)\right)$ be a solution of (1.6) and let

$$
\beta_{i}(\tau)=\int_{\mathbb{R}^{3}} p_{i}(X) \cdot W(\tau, X) d X .
$$

Then, for all $\tau \in\left[\tau_{0}, T\right]$,

$$
\beta_{i}(\tau)=b_{i} e^{-\tau}
$$

where $b_{i}=\int_{\mathbb{R}^{3}} p_{i}(X) \cdot W_{0}(X) d X$.

Proof. The proof of this lemma is made formally, assuming that every quantity that we consider is well defined. Actually, in the remaining of this article, we will work with regularized solutions for which the next computations are rigorous. In order to get (2.5), we only have to show that $\beta_{i}$ satisfies

$$
\partial_{\tau} \beta_{i}(\tau)=-\beta_{i}(\tau) \text {. }
$$

Performing the $L^{2}$-scalar product of the first equality of (1.6) with $p_{i}$, we obtain $(2.7)$

$$
\begin{aligned}
\partial_{\tau} \beta_{i}(\tau)=\alpha e^{-\tau}\left(p_{i}, \partial_{\tau} \Delta W\right)_{L^{2}}-\alpha e^{-\tau}\left(p_{i}, \Delta W\right)_{L^{2}}+\left(p_{i}, \mathcal{L}(W)\right)_{L^{2}} \\
\quad+\left(p_{i}, \operatorname{curl}\left(\left(W-\alpha e^{-\tau} \Delta W\right) \wedge U\right)\right)_{L^{2}}-\alpha e^{-\tau}\left(p_{i}, \Delta W+\frac{X}{2} \cdot \nabla \Delta W\right)_{L^{2}} .
\end{aligned}
$$

Integrating several times by parts, it is easy to check that

$$
\alpha e^{-\tau}\left(p_{i}, \partial_{\tau} \Delta W\right)_{L^{2}}=\alpha e^{-\tau}\left(p_{i}, \Delta W\right)_{L^{2}}=\alpha e^{-\tau}\left(p_{i}, \Delta W+\frac{X}{2} \cdot \nabla \Delta W\right)_{L^{2}}=0,
$$

and the equality (2.3) implies

$$
\left(p_{i}, \mathcal{L}(W)\right)_{L^{2}}=\left(\mathcal{L}^{*} p_{i}, W\right)_{L^{2}}=-\left(p_{i}, W\right)_{L^{2}}=-\beta_{i}(\tau) .
$$

Thus, integrating by parts and recalling that curl $p i=e_{i}$, one has

$$
\partial_{\tau} \beta_{i}(\tau)=-\beta_{i}(\tau)+\int_{\mathbb{R}^{3}} e_{i} \cdot\left(\left(W(X)-\alpha e^{-\tau} \Delta W(X)\right) \wedge U(X)\right) d X .
$$

It remains to show that the last term of the right hand size of (2.8) vanishes. Noticing that $W=\operatorname{curl} U$, an easy computation shows, for $i \in\{1,2,3\}$,

$$
\begin{aligned}
& \left(U(X) \wedge\left(W(X)-\alpha e^{-\tau} \Delta W(X)\right)\right)_{i}= \\
& \frac{1}{2} \partial_{i}\left(|U|^{2}\right)-U . \nabla U_{i}-\alpha e^{-\tau}\left(U . \partial_{i} \Delta U-U . \nabla \Delta U_{i}\right) .
\end{aligned}
$$

Thus, using the divergence free property of $U$ and integrating by parts, we get

$$
\int_{\mathbb{R}^{3}} e_{i} \cdot\left(U(X) \wedge\left(W(X)-\alpha e^{-\tau} \Delta W(X)\right)\right) d X=-\alpha e^{-\tau} \int_{\mathbb{R}^{3}} U(X) . \partial_{i} \Delta U(X) d X .
$$

Another integration by parts yields

$$
\int_{\mathbb{R}^{3}} e_{i} \cdot\left(U(X) \wedge\left(W(X)-\alpha e^{-\tau} \Delta W(X)\right)\right) d X=\frac{\alpha}{2} e^{-\tau} \int_{\mathbb{R}^{3}} \partial_{i}\left(|\nabla U(X)|^{2}\right) d X=0,
$$

and thus we obtain (2.6). 
We can now state the main theorem of this paper, which shows in particular that the first order asymptotic profile of a solution $W$ in $\mathbb{H}^{2}(4)$ of (1.6) is the same as the first order asymptotic profile obtained for the Navier-Stokes equations.

THEOREM 2.2. Let $\theta$ be a fixed constant such that $1<\theta<\frac{3}{2}$ and $W_{0} \in \mathbb{H}^{2}(4)$. There exist two positive constants $\gamma_{0}=\gamma_{0}(\alpha)$ and $T_{0}=T_{0}(\alpha) \geq 1$ such that if $T \geq T_{0}$ and there exists a positive constant $\gamma \leq \gamma_{0}$ such that

$\left\|W_{0}\right\|_{L^{2}(4)}^{2}+\left\|\nabla W_{0}\right\|_{L^{2}}^{2}+\alpha e^{-\tau_{0}}\left\|\Delta W_{0}\right\|_{L^{2}}^{2}+\alpha^{2} e^{-2 \tau_{0}}\left\||X|^{4} \Delta W_{0}\right\|_{L^{2}}^{2} \leq \gamma\left(\frac{3}{2}-\theta\right)^{2}$,

where $\tau_{0}=\log (T)$,

then there exist a unique solution $W \in C^{0}\left(\left[\tau_{0},+\infty\right), \mathbb{H}^{2}(4)\right)$ to the system (1.6) and a positive constant $C=C\left(\alpha, T_{0}\right)$ such that

$$
\left\|\left(I-\alpha e^{-\tau} \Delta\right)\left(W(\tau)-e^{-\tau} \sum_{i=1}^{3} b_{i} f_{i}\right)\right\|_{L^{2}(4)} \leq C \gamma^{1 / 2}\left(\frac{3}{2}-\theta\right) e^{-\theta \tau},
$$

where $b_{i}=\int_{\mathbb{R}^{3}} p_{i}(X) \cdot W_{0}(X) d X$.

In the classical variables, the next corollary is deduced from Theorem 2.2.

COROllary 2.1. Let $\theta$ be a constant such that $1<\theta<\frac{3}{2}, w_{0} \in \mathbb{H}^{2}(4)$ and $b_{i}=\frac{1}{T} \int_{\mathbb{R}^{3}} p_{i}(x) \cdot w_{0}(x) d x$. There exist $\gamma_{0}=\gamma_{0}(\alpha)>0$ and $T_{0}=T_{0}(\alpha, \theta) \geq 1$ such that if there exist $T \geq T_{0}$ and $\gamma \leq \gamma_{0}$ such that

$$
\begin{aligned}
T^{1 / 2}\left\|w_{0}\right\|_{L^{2}}^{2}+T^{-7 / 2}\left\||x|^{4} w_{0}\right\|_{L^{2}}^{2}+T^{3 / 2}\left\|\nabla w_{0}\right\|_{L^{2}}^{2} \\
\quad+\alpha T^{3 / 2}\left\|\Delta w_{0}\right\|_{L^{2}}^{2}+\alpha^{2} T^{-3 / 2}\left\||x|^{4} \Delta w_{0}\right\|_{L^{2}}^{2} \leq \gamma\left(\frac{3}{2}-\theta\right)^{2},
\end{aligned}
$$

then there exists a unique solution $w \in C^{0}\left([0,+\infty), \mathbb{H}^{2}(4)\right)$ to the system (1.2) such that, for all $1 \leq p \leq 2$, the following inequality holds

$$
\begin{aligned}
& \left\|(I-\alpha \Delta)\left(w(t)-\sum_{i=1}^{3} \frac{b_{i}}{(t+T)^{2}} f_{i}\left(\frac{x}{\sqrt{t+T}}\right)\right)\right\|_{L^{p}} \\
& \leq C \gamma^{1 / 2}\left(\frac{3}{2}-\theta\right)(t+T)^{-1-\theta+\frac{3}{2 p}}
\end{aligned}
$$

where $C=C\left(\alpha, T_{0}\right)$ is a positive constant. Besides, for all $1 \leq p \leq+\infty$, one has

$$
\left\|w(t)-\sum_{i=1}^{3} \frac{b_{i}}{(t+T)^{2}} f_{i}\left(\frac{x}{\sqrt{t+T}}\right)\right\|_{L^{p}} \leq C \gamma^{1 / 2}\left(\frac{3}{2}-\theta\right)(t+T)^{-1-\theta+\frac{3}{2 p}} .
$$

Let $u$ be the divergence free vector field obtained from $w$ through the Biot-Savart law. For all $\frac{3}{2} \leq p \leq+\infty$, one has

$$
\left\|u(t)-\sum_{i=1}^{3} \frac{b_{i}}{(t+T)^{3 / 2}} v_{i}\left(\frac{x}{\sqrt{t+T}}\right)\right\|_{L^{p}} \leq C \gamma^{1 / 2}\left(\frac{3}{2}-\theta\right)(t+T)^{-\frac{1}{2}-\theta+\frac{3}{2 p}},
$$

where $v_{i}$ is obtained from $f_{i}$ via the Biot-Savart law. 
Theorem 2.2 and Corollary 2.1 describe the first order asymptotic profiles of the solutions of the second grade fluids equations. In particular, they show that these solutions behave asymptotically like the self-similar solutions to the heat equation given by

$$
(t, x) \longrightarrow \sum_{i=1}^{3} \frac{b_{i}}{(t+T)^{2}} f_{i}\left(\frac{x}{\sqrt{t+T}}\right) .
$$

In addition, since the same result has been shown in [17] for the Navier-Stokes equations, it shows that, at the first order, the second grade fluids behave asymptotically like Newtonian fluids.

REMARK 2.3. We emphasize that the convergence results of Theorem 2.2 and Corollary 2.1 allow to choose the rate of convergence as close as wanted to the optimal one, provided the initial data are small enough in $\mathbb{H}^{2}(4)$. In dimension 2, the rate of convergence of the results of Jaffal-Mourtada in [23] cannot be better than $e^{-\tau / 4}$, whereas the optimal one is $e^{-\tau / 2}$. In Section 4 , we will see that the method used in the present paper to make estimates on the solutions of (1.6) in Sobolev spaces of negative order differs from the one used in $[\mathbf{2 3}]$, which is the reason why we are able to obtain a better rate of convergence.

We prove Theorem 2.2 in several steps. First, in Section 3, we introduce a new system that is close to (1.6), but which contains the regularizing term $\varepsilon \Delta^{2} W$, with $\varepsilon$ a small positive constant that is devoted to tend to 0 . Due to this regularizing term, we are able, through a semi-group method, to show the existence of local solutions to the regularized system. In a second time, in Section 4 we perform energy estimates on these approximate solutions, and show that these ones are global in time and satisfy the inequality (2.11). Then, in Section 5, we pass to the limit when $\varepsilon$ tends to 0 and show that the approximate solutions converge to a global weak solution of (1.6) which satisfies the inequality (2.11). Finally, in order to show that every solution whose initial data satisfy the assumption (2.10) converge to his first order asymptotic profile, we show the uniqueness of the weak solutions of (1.2) belonging to $C^{0}\left([0,+\infty), \mathbb{H}^{2}(4)\right)$.

Biot-Savart law: We recall some properties of the Biot-Savart law. Let $w$ be a given divergence free vector field of $\mathbb{R}^{3}$, the Biot-Savart law gives a divergence free vector field $u$ such that curl $u=w$. It is given by

$$
u(x)=-\frac{1}{4 \pi} \int_{\mathbb{R}^{3}} \frac{(x-y) \wedge w(y)}{|x-y|^{3}} d y .
$$

In particular, the scaled variables (1.5) preserves the Biot-Savart law. Indeed, if $u$ is obtained from $w$ via the Biot-Savart law and $W$ is $w$ expressed into scaled variables, then the divergence free vector field $U$ obtained from $W$ through the Biot-Savart law is $u$ expressed in scaled variables. The next lemma gives some estimates on vector fields obtained by $(2.16)$, in various functions spaces.

LEMMA 2.4. Let $w$ be a divergence free vector field of $\mathbb{R}^{3}$ and $u$ be the velocity field obtained from $w$ via the Biot-Savart law (2.16).

(a) Assume that $1<p<3$, $\frac{3}{2}<q<\infty$ and $\frac{1}{q}=\frac{1}{p}-\frac{1}{3}$. If $w \in L^{p}\left(\mathbb{R}^{3}\right)^{3}$, then $u \in L^{q}\left(\mathbb{R}^{3}\right)^{3}$, and there exists $C>0$ such that

$$
\|u\|_{L^{q}} \leq C\|w\|_{L^{p}} .
$$


(b) Assume that $1 \leq p<3<q \leq \infty$, and define $\eta \in(0,1)$ by the relation $\frac{1}{3}=\frac{\eta}{p}+\frac{(1-\eta)}{q}$. If $w \in L^{p}\left(\mathbb{R}^{3}\right)^{3} \cap L^{q}\left(\mathbb{R}^{3}\right)^{3}$, then $u \in L^{\infty}\left(\mathbb{R}^{3}\right)^{3}$ and there exists $C>0$ such that

$$
\|u\|_{L^{\infty}} \leq C\|w\|_{L^{p}}^{\eta}\|w\|_{L^{q}}^{1-\eta} .
$$

(c) Assume that $1<p<\infty$. If $w \in L^{p}\left(\mathbb{R}^{3}\right)^{3}$, then $\nabla u \in L^{p}\left(\mathbb{R}^{3}\right)^{9}$ and there exists $C>0$ such that

$$
\|\nabla u\|_{L^{p}} \leq C\|w\|_{L^{p}} .
$$

This lemma is proved in $[\mathbf{1 7}]$ and will be very useful when making estimates on the solutions of (1.6).

\section{Approximate solutions}

In this section, we introduce a new system that is close to (1.2), but contains the regularizing term $\varepsilon \Delta^{2} w$, where $\varepsilon$ is a small positive constant. We introduce such a system in order to get smooth solutions, for which we are able to perform estimates in $\mathbb{H}^{2}(4)$ and obtain the inequality (2.11). In Section 5 , we pass to the limit when $\varepsilon$ goes to 0 and show that the limit of the solution of the regularized system is a weak solutions of the system (1.6), which also satisfies the inequality (2.11). We introduce the following regularized system, given by

$$
\begin{aligned}
& \partial_{t}\left(w_{\varepsilon}-\alpha \Delta w_{\varepsilon}\right)+\varepsilon \Delta^{2} w_{\varepsilon}-\Delta w_{\varepsilon}+\operatorname{curl}\left(\left(w_{\varepsilon}-\alpha \Delta w_{\varepsilon}\right) \wedge u_{\varepsilon}\right)=0, \\
& \operatorname{div} u_{\varepsilon}=\operatorname{div} w_{\varepsilon}=0, \\
& w_{\varepsilon \mid t=0}=w_{0} .
\end{aligned}
$$

The next theorem shows that, for every $w_{0} \in \mathbb{H}^{2}(4)$, there exists a unique local solution to (3.1) belonging to $\mathbb{H}^{2}(4)$, which is smooth enough to perform the estimates of Section 4.

TheOREM 3.1. Let $\varepsilon>0$ and $w_{0} \in \mathbb{H}^{2}(4)$. There exists $t_{\varepsilon}>0$ and a unique solution $w_{\varepsilon}$ to the system (3.1) defined on the time interval $\left[0, t_{\varepsilon}\right)$ such that

$$
w_{\varepsilon} \in C^{1}\left(\left(0, t_{\varepsilon}\right), \mathbb{H}^{1}(4)\right) \cap C^{0}\left(\left[0, t_{\varepsilon}\right), \mathbb{H}^{2}(4)\right) \cap C^{0}\left(\left(0, t_{\varepsilon}\right), \mathbb{H}^{3}(4)\right) .
$$

Proof. To get this result, one defines $w_{\varepsilon, \mu}(t, x)=w_{\varepsilon}\left(t, \frac{x}{\mu}\right)$, where $\mu>0$. This change of variables enables us to show the existence of solutions to the system (3.1) without restrictions on the size of the parameter $\alpha$. We define $u_{\varepsilon, \mu}$ obtained from $w_{\varepsilon, \mu}$ by the Biot-Savart law (2.16). It is easy to check that $u_{\varepsilon, \mu}(t, x)=$ $\mu u_{\varepsilon}\left(t, \frac{x}{\mu}\right)$. In order to show the existence of a unique solution to (3.1), we will prove that there exists a unique solution to the system

$$
\begin{aligned}
& \partial_{t}\left(w_{\varepsilon, \mu}-\alpha \mu^{2} \Delta w_{\varepsilon, \mu}\right)-\varepsilon \mu^{4} \Delta^{2} w_{\varepsilon, \mu}-\mu^{2} \Delta w_{\varepsilon, \mu}+ \\
& \operatorname{curl}\left(\left(w_{\varepsilon, \mu}-\alpha \mu^{2} \Delta w_{\varepsilon, \mu}\right) \wedge u_{\varepsilon, \mu}\right)=0 \\
& \operatorname{div} w_{\varepsilon, \mu}=\operatorname{div} u_{\varepsilon, \mu}=0 \\
& w_{\varepsilon, \mu \mid t=0}=w_{0}\left(\frac{x}{\mu}\right) \in \mathbb{H}^{2}(4) .
\end{aligned}
$$

We define now $z_{\varepsilon}(t, x)=q(x) w_{\varepsilon, \mu}(t, x)$, where $q(x)=\left(1+|x|^{4}\right)$. In particular, if $w_{\varepsilon, \mu} \in \mathbb{L}^{2}(4)$, then $z_{\varepsilon} \in \mathcal{H}$, where

$$
\mathcal{H}=\left\{z \in L^{2}\left(\mathbb{R}^{3}\right)^{3}: \operatorname{div}\left(q^{-1} z\right)=0\right\} .
$$

For later use, we define, for $s \geq 0$, 


$$
\mathcal{H}^{s}=\mathcal{H} \cap H^{s}\left(\mathbb{R}^{3}\right)^{3}, \quad \text { and } \quad \mathcal{H}^{-s}=\left(\mathcal{H}^{s}\right)^{\prime},
$$

where $\left(\mathcal{H}^{s}\right)^{\prime}$ denotes the dual space of $\mathcal{H}^{s}$.

We equip $\mathcal{H}^{s}$ with the classical $H^{s}$ Sobolev norm, which makes $\mathcal{H}^{s}$ complete. From the system (3.2), we deduce the following one, that we solve in $z_{\varepsilon}$,

$$
\begin{aligned}
& \partial_{\tau}\left(z_{\varepsilon}-\alpha \mu^{2} \Delta z_{\varepsilon}-\alpha \mu^{2} q \Delta q^{-1} z_{\varepsilon}-2 \alpha \mu^{2} q \nabla q^{-1} \cdot \nabla z_{\varepsilon}\right)+\varepsilon \mu^{4} \Delta^{2} z_{\varepsilon}=F\left(x, z_{\varepsilon}\right), \\
& \operatorname{div}\left(q^{-1} z_{\varepsilon}\right)=0 \\
& z_{\varepsilon \mid t=0}(x)=z_{0}(x) \in \mathcal{H}^{2}
\end{aligned}
$$

where

$$
\begin{aligned}
& F\left(x, z_{\varepsilon}\right)=-\varepsilon \mu^{4} q \Delta^{2}\left(q^{-1} z_{\varepsilon}\right)+\mu^{2} q \Delta\left(q^{-1} z_{\varepsilon}\right) \\
& +q \operatorname{curl}\left(\left(q^{-1} z_{\varepsilon}-\mu^{2} \alpha_{1} \Delta\left(q^{-1} z_{\varepsilon}\right)\right) \wedge u_{\varepsilon, \mu}\right) .
\end{aligned}
$$

The system (3.3) is actually autonomous. Indeed, one can recover $u_{\varepsilon, \mu}$ by the BiotSavart law (2.16) applied to $q^{-1} z_{\varepsilon}$. To show the existence of solutions to (3.1) in $\mathbb{H}^{1}(4)$, it suffices to show the existence of solutions to (3.3) in $\mathcal{H}^{1}$, for data belonging to $\mathcal{H}^{2}$.

We set two linear differential operators $B: D(B)=\mathcal{H}^{1} \rightarrow \mathcal{H}^{-1}$ and $D: D(D)=$ $\mathcal{H} \rightarrow \mathcal{H}^{-1}$, given by

$$
\begin{aligned}
& B=\alpha \mu^{2} q \Delta q^{-1}+\alpha \mu^{2} \Delta, \\
& D=\alpha \mu^{2} q \nabla q^{-1} \cdot \nabla .
\end{aligned}
$$

Via Lax-Milgram theorem, we show now that if $\mu$ is sufficiently small with respect to $\alpha$, the operator $(I-B-D)$ is invertible. In order to do that, we define the bilinear form on $\mathcal{H}^{1} \times \mathcal{H}^{1}$, given by

$$
a(u, v)=(u, v)_{L^{2}}+\alpha \mu^{2}(\nabla u, \nabla v)_{L^{2}}-\alpha \mu^{2}\left(q \Delta q^{-1} u, v\right)_{L^{2}}-2 \alpha \mu^{2}\left(q \nabla q^{-1} \cdot \nabla u, v\right)_{L^{2}} .
$$

Since $q \Delta q^{-1}$ and $q \nabla q^{-1}$ are bounded on $\mathbb{R}^{3}$, the bilinear form $a$ is continuous on $\mathcal{H}^{1}$. We now show, taking $\mu$ small enough, that $a$ is also coercive on $\mathcal{H}^{1}$. Indeed, integrating by parts and using Hölder and Young inequalities, we have

$$
a(u, u) \geq\left(1-\alpha \mu^{2} \sup _{x \in \mathbb{R}^{3}}\left(q \Delta q^{-1}\right)+\alpha \mu^{2} \inf _{x \in \mathbb{R}^{3}}\left(\operatorname{div}\left(q \nabla q^{-1}\right)\right)\right)\|u\|_{L^{2}}^{2}+\alpha \mu^{2}\|\nabla u\|_{L^{2}}^{2} .
$$

Thus, if we take $\mu$ sufficiently small, we get

$$
a(u, u) \geq C(\alpha, \mu)\|u\|_{H^{1}}^{2},
$$

where $C(\alpha, \mu)$ is a positive constant depending on $\alpha$ and $\mu$.

The classical Lax-Milgram theorem enables us to define $(I-B-D)^{-1}$ from $\mathcal{H}^{-1}$ to $\mathcal{H}^{1}$. We define the linear differential operator $A: D(A)=\mathcal{H}^{3} \rightarrow \mathcal{H}^{1}$ given by

$$
A=\varepsilon \mu^{4}(I-B-D)^{-1} \Delta^{2} .
$$

We can rewrite the system (3.3) as follows:

$$
\begin{aligned}
& \partial_{\tau} z_{\varepsilon}+A z_{\varepsilon}=(I-B-D)^{-1} F\left(x, z_{\varepsilon}\right), \\
& z_{\varepsilon \mid t=0}=z_{0} .
\end{aligned}
$$


In order to show the existence of solutions to such a system, we use, like in [23], a semi-group method. First, we show that $-A$ generates an analytic semi-group on $\mathcal{H}^{1}$ which is equivalent as $A$ is sectorial on $\mathcal{H}^{1}$. We decompose $A$ as follows:

$$
\begin{aligned}
A & =\varepsilon \mu^{4}(I d-B-D)^{-1} \Delta^{2} \\
& =\varepsilon \mu^{4}(I d-B)^{-1} \Delta^{2}+\varepsilon \mu^{4}(I d-B-D)^{-1} D(I d-B)^{-1} \Delta^{2} \\
& =J+R,
\end{aligned}
$$

where

$$
\begin{aligned}
& J=I d+\varepsilon \mu^{4}(I d-B)^{-1} \Delta^{2}, \\
& R=-I d+\varepsilon \mu^{4}(I d-B-D)^{-1} D(I d-B)^{-1} \Delta^{2} .
\end{aligned}
$$

We first show that $J$ is sectorial. We will see later that $R$ satisfies properties that enable to conclude that $A$ is sectorial if $J$ is sectorial. Taking $\mu$ sufficiently small compared to $\alpha$, it is easy, arguing like we did to invert $(I-B-D)$, to show that $(I-B)^{-1}$ is well defined from $\mathcal{H}^{-1}$ to $\mathcal{H}^{1}$. Consequently, the operator $J$ is well defined from $\mathcal{H}^{3}$ to $\mathcal{H}^{1}$. We define now the bilinear form $j$ on $\mathcal{H}^{2} \times \mathcal{H}^{2}$ associated to $J$. To this end, we introduce a $H^{1}$-scalar product which is adapted to $J$. We define

$$
\langle u, v\rangle_{H^{1}}=\left(\left(1-\alpha \mu^{2} q \Delta q^{-1}\right) u, v\right)_{L^{2}}+\alpha \mu^{2}(\nabla u, \nabla v)_{L^{2}} .
$$

If $\mu$ is sufficiently small, $\langle., .\rangle_{H^{1}}$ is a scalar product on $\mathcal{H}^{1}$. In particular, if $u \in \mathcal{H}^{2}$ and $v \in \mathcal{H}^{1}$, one has

$$
\langle u, v\rangle_{H^{1}}=((I-B) u, v)_{L^{2}} .
$$

Via this product, we define

$$
j(u, v)=\langle u, v\rangle_{H^{1}}+\varepsilon \mu^{4}(\Delta u, \Delta v) .
$$

In particular, if $u \in \mathcal{H}^{3}$ and $v \in \mathcal{H}^{1}$, one has

$$
j(u, v)=\langle J u, v\rangle_{H^{1}} .
$$

The bilinear form $j$ is obviously continuous on $\mathcal{H}^{2} \times \mathcal{H}^{2}$. Furthermore, if $\mu$ is small enough, it is also coercive on $\mathcal{H}^{2}$. Indeed,

$$
\begin{aligned}
j(u, u) & \geq C(\alpha, \mu)\|u\|_{H^{1}}^{2}+\varepsilon \mu^{4}\|\Delta u\|_{L^{2}}^{2} \\
& \geq C(\alpha, \mu, \varepsilon)\|u\|_{H^{2}}^{2} .
\end{aligned}
$$

Thus $j$ is continuous and coercive on $\mathcal{H}^{2}$ and consequently $J$ is sectorial on $\mathcal{H}^{1}$, that is equivalent to say that $-J$ generates an analytic semi-group on $\mathcal{H}^{1}$. Furthermore, we can check that $R$ is continuous from $\mathcal{H}^{2}$ to $\mathcal{H}^{1}$, and we have

$$
\|R u\|_{H^{1}} \leq C(\alpha, \mu, \varepsilon)\|u\|_{H^{2}} .
$$

Using the coerciveness of $j$, we get, for all $u \in \mathcal{H}^{3}$,

$$
\begin{aligned}
\|R u\|_{H^{1}}^{2} & \leq C(\alpha, \mu, \varepsilon) j(u, u) \\
& \leq C(\alpha, \mu, \varepsilon)\langle J u, u\rangle_{H^{1}} \\
& \leq C\|J u\|_{H^{1}}\|u\|_{H^{1}} .
\end{aligned}
$$

Applying the Young inequality, we obtain, for all $\delta>0$

$$
\|R u\|_{H^{1}}^{2} \leq \delta\|J u\|_{H^{1}}^{2}+C\|u\|_{H^{1}}^{2} \text {, for all } u \in \mathcal{H}^{3} .
$$


From a classical result that we can find in the book of D. Henry [22], it implies that $J+R$ is sectorial on $\mathcal{H}^{1}$.

To achieve this proof, we check that $A^{-1} F(x, v)$ is locally Lipschitz in $v \in \mathcal{H}^{1}$ on the bounded sets of $\mathcal{H}^{2}$. According to [27, section 6.3] and [22, chapter 3], we finally get Theorem 3.1 .

\section{Energy estimates}

In this section, we perform several energy estimates on the solution of the system (3.1) given by Theorem 3.1. We consider a fixed positive constant $\theta$ such that $1<\theta<\frac{3}{2}$, which is the rate of convergence of Theorem 2.2. Let $T$ be a positive constant which will be made more precise later and that we assume, without loss of generality, to be such that $T \geq 1$. We consider $W_{\varepsilon}$ the divergence free vector field obtained from $w_{\varepsilon}$ via the change of variables (1.5). According to Theorem 3.1 , there exists a maximal time $\tau_{\varepsilon}$ such that $W_{\varepsilon}$ belongs to $C^{1}\left(\left(\tau_{0}, \tau_{\varepsilon}\right), \mathbb{H}^{1}(4)\right) \cap$ $C^{0}\left(\left(\tau_{0}, \tau_{\varepsilon}\right), \mathbb{H}^{3}(4)\right)$, where $\tau_{0}=\log (T)$. A short computation shows that $W_{\varepsilon}$ is the solution of the system

$$
\begin{aligned}
& \partial_{\tau}\left(W_{\varepsilon}-\alpha e^{-\tau} \Delta W_{\varepsilon}\right)+\varepsilon e^{-\tau} \Delta^{2} W_{\varepsilon}-\mathcal{L}\left(W_{\varepsilon}\right)+\operatorname{curl}\left(\left(W_{\varepsilon}-\alpha e^{-\tau} \Delta W_{\varepsilon}\right) \wedge U_{\varepsilon}\right) \\
& +\alpha e^{-\tau} \Delta W_{\varepsilon}+\alpha e^{-\tau} \frac{X}{2} \cdot \nabla \Delta W_{\varepsilon}=0, \\
& \operatorname{div} U_{\varepsilon}=\operatorname{div} W_{\varepsilon}=0, \\
& W_{\varepsilon \mid \tau=\tau_{0}}=W_{0},
\end{aligned}
$$

where we recall that

$$
\mathcal{L}\left(W_{\varepsilon}\right)=W_{\varepsilon}+\Delta W_{\varepsilon}+\frac{X}{2} \cdot \nabla W_{\varepsilon} .
$$

In this section, we obtain several energy estimates in various functions spaces. More precisely, assuming that $T$ is large enough and $W_{0}$ is small enough in $\mathbb{H}^{2}(4)$, we show that the solution of (4.1) stays bounded in time in those energy spaces and is consequently global in time. In addition, we obtain the inequality $(2.11)$ for $W_{\varepsilon}$. The method to reach this aim is based on the construction of an energy functional $E$ such that

$$
E(\tau) \sim\left\|W_{\varepsilon}(\tau)-e^{-\tau} \sum_{i=1}^{3} b_{i} f_{i}\right\|_{H^{2}(4)}^{2}, \text { for all } \tau \geq \log (T),
$$

and there exists a positive constant $C$ such that, for all $\tau \geq \log (T)$,

$$
\partial_{\tau} E(\tau)+2 \theta E(\tau) \leq C e^{-3 \tau},
$$

where $b_{i}=\int_{\mathbb{R}^{3}} p_{i}(X) . W_{0}(X) d X$ and $\left\{f_{1}, f_{2}, f_{3}\right\}$ is the basis of the eigenspace of $\mathcal{L}$ associated to the eigenvalue -1 , given by (2.1). Through the Gronwall Lemma, the inequality (4.2) allows to get the inequality (2.11) for $W_{\varepsilon}$ and to conclude that $W_{\varepsilon}$ is global in time.

We set $\Omega_{\infty}=\sum_{i=1}^{3} b_{i} f_{i}$. The decomposition (2.4) becomes

$$
W_{\varepsilon}(\tau)=e^{-\tau} \Omega_{\infty}+R_{\varepsilon}(\tau)
$$


A short computation shows that $R_{\varepsilon}$ satisfies the equality (4.4)

$$
\begin{aligned}
& \partial_{\tau}\left(R_{\varepsilon}-\alpha e^{-\tau} \Delta R_{\varepsilon}\right)+\varepsilon e^{-\tau} \Delta^{2} R_{\varepsilon}-\mathcal{L}\left(R_{\varepsilon}\right)+\operatorname{curl}\left(\left(W_{\varepsilon}-\alpha e^{-\tau} \Delta W_{\varepsilon}\right) \times U_{\varepsilon}\right) \\
& +\alpha e^{-\tau} \Delta R_{\varepsilon}+\alpha e^{-\tau} \frac{X}{2} \cdot \nabla \Delta R_{\varepsilon}+3 \alpha e^{-2 \tau} \Delta \Omega_{\infty}+\varepsilon e^{-2 \tau} \Delta^{2} \Omega_{\infty}=0
\end{aligned}
$$

In this section, we assume that $W_{0}$ satisfies the condition (2.10) of Theorem 2.2 for some positive constant $\gamma$. We also set $M$ to be a positive constant such that $M \geq 2$ which will be made more precise later. Let $\tau_{\varepsilon}^{*}, \tau_{0}<\tau_{\varepsilon}^{*} \leq \tau_{\varepsilon}$ be the largest positive time such that, for all $\tau \in\left[\tau_{0}, \tau_{\varepsilon}^{*}\right)$,

$$
\begin{aligned}
\left\|W_{\varepsilon}(\tau)\right\|_{L^{2}(4)}^{2}+\left\|\nabla W_{\varepsilon}(\tau)\right\|_{L^{2}}^{2}+ & \alpha e^{-\tau}\left\|\Delta W_{\varepsilon}(\tau)\right\|_{L^{2}}^{2} \\
& \quad+\alpha^{2} e^{-2 \tau}\left\||X|^{4} \Delta W_{\varepsilon}(\tau)\right\|_{L^{2}}^{2} \leq M \gamma\left(\frac{3}{2}-\theta\right)^{2} .
\end{aligned}
$$

Since $R_{\varepsilon}$ belongs to $C^{0}\left(\left[\tau_{0}, \tau_{\varepsilon}\right), \mathbb{H}^{2}(4)\right)$, the time $\tau_{\varepsilon}^{*}$ is well defined. The next lemma, which is a consequence of (4.5), establishes an inequality on the $\mathbb{H}^{2}(4)$-norm of $R_{\varepsilon}$.

LEMma 4.1. Let $W_{\varepsilon} \in C^{0}\left(\left[\tau_{0}, \tau_{\varepsilon}^{*}\right), \mathbb{H}^{2}(4)\right)$ satisfying the condition (4.5) and $R_{\varepsilon}=W_{\varepsilon}-e^{-\tau} \Omega_{\infty}$. There exists a positive constant $C$ such that, for all $\tau \in\left[\tau_{0}, \tau_{\varepsilon}^{*}\right)$, (4.6)

$$
\begin{aligned}
|b|^{2}+\left\|R_{\varepsilon}(\tau)\right\|_{L^{2}(4)}^{2}+\left\|\nabla R_{\varepsilon}(\tau)\right\|_{L^{2}}^{2}+\alpha e^{-\tau}\left\|\Delta R_{\varepsilon}(\tau)\right\|_{L^{2}}^{2} & \\
& +\alpha^{2} e^{-2 \tau}\left\||X|^{4} \Delta R_{\varepsilon}(\tau)\right\|_{L^{2}}^{2} \leq C M \gamma\left(\frac{3}{2}-\theta\right)^{2} .
\end{aligned}
$$

Proof. To prove the inequality (4.6), we notice that, for all $i \in\{1,2,3\}$,

$$
\begin{aligned}
\left|b_{i}\right| & \leq \int_{\mathbb{R}^{2}}|X|\left|W_{0}\right| d X \\
& \leq\left(\int_{\mathbb{R}^{2}} \frac{1}{\left(1+|X|^{2}\right)^{3}} d X\right)^{1 / 2}\left(\int_{\mathbb{R}^{2}}\left(1+|X|^{2}\right)^{3}|X|^{2}\left|W_{0}\right|^{2} D X\right)^{1 / 2} \\
& \leq C\left\|W_{0}\right\|_{L^{2}(4)} .
\end{aligned}
$$

Thus, recalling that $R_{\varepsilon}=W_{\varepsilon}-e^{-\tau} \sum_{i=1}^{3} b_{i} f_{i}$ and taking into account (2.10), we obtain (4.6).

For the sake of simplicity, we assume in this section that $\gamma \leq 1$ and $\alpha e^{-\tau_{0}} \leq 1$. In what follows, $C$ denotes a positive constant, which eventually depends on $\alpha$, and which can change from one line to another. To simplify the notations, we also note $R$ instead of $R_{\varepsilon}, W$ instead of $W_{\varepsilon}$ and $U$ instead of $U_{\varepsilon}$.

4.1. Estimates in $H^{-(\theta+2)}\left(\mathbb{R}^{3}\right)$. In this section, we perform an estimate of $R_{\varepsilon}$ in the space $H^{-(\theta+2)}\left(\mathbb{R}^{3}\right)$ on the time interval $\left[\tau_{0}, \tau_{\varepsilon}^{*}\right)$. This is motivated by the fact that, in the $H^{1}$-estimate that we establish below, the term $\left\|R_{\varepsilon}\right\|_{L^{2}}^{2}$ takes place in the right hand side of the inequality (4.2). To absorb this term, we look for an estimate in the homogeneous Sobolev space $\dot{H}^{-(\theta+2)}\left(\mathbb{R}^{3}\right)$. Combined with the other energy estimates, it gives an estimate in the classical Sobolev space $H^{-(\theta+2)}\left(\mathbb{R}^{3}\right)$. 
Notice that the constant $\theta+2$ is chosen in order to obtain the term $2 \theta E$ in the inequality (4.2). In [23], the choice of the Sobolev space of negative order do not depend on $\theta$, that is why the rate of convergence obtained in [23] cannot be taken as close as wanted to the optimal one. In order to perform this energy estimate, we define, for $s \in \mathbb{R}$, the operator

$$
(-\Delta)^{-s} u=\overline{\mathcal{F}}\left(\frac{1}{|\xi|^{4 s}} \widehat{u}\right),
$$

where $\widehat{u}$ is the Fourier transform of $u$, given by

$$
\widehat{u}(\xi)=\int_{\mathbb{R}^{3}} e^{-i x \cdot \xi} u(x) d x,
$$

and $\overline{\mathcal{F}}$ is the inverse Fourier transform.

We are allowed to consider $(-\Delta)^{-\left(\frac{\theta}{2}+1\right)} R_{\varepsilon}$ by the lemma

LEMmA 4.2. Let $u \in L^{2}(4)$ such that $\int_{\mathbb{R}^{3}} u(x) d x=0$.

(1) If $\int_{\mathbb{R}^{3}} x_{i} u(x) d x=0$ for every $i \in\{1,2,3\}$, then, for all $0 \leq s<\frac{7}{4}$, $(-\Delta)^{-s} u \in L^{2}\left(\mathbb{R}^{3}\right)$ and there exists a positive constant $C$ such that

$$
\left\|(-\Delta)^{-s} u\right\|_{L^{2}} \leq \frac{C}{\sqrt{7-4 s}}\|u\|_{L^{2}(4)} .
$$

(2) For all $0 \leq s<\frac{7}{4}$, $(-\Delta)^{-s} \nabla u \in L^{2}\left(\mathbb{R}^{3}\right)^{3}$ and there exists a positive constant $C$ such that

$$
\left\|(-\Delta)^{-s} \nabla u\right\|_{L^{2}} \leq \frac{C}{\sqrt{7-4 s}}\|u\|_{L^{2}(3)} .
$$

Proof. Using Fourier variables, we get

$$
\begin{aligned}
\left\|(-\Delta)^{-s} u\right\|_{L^{2}}^{2} & =\frac{1}{(2 \pi)^{3}} \int_{\mathbb{R}^{2}} \frac{1}{|\xi|^{4 s}}|\widehat{u}(\xi)|^{2} d \xi \\
& \leq \frac{1}{(2 \pi)^{3}} \int_{|\xi| \leq 1} \frac{1}{|\xi|^{4 s}}|\widehat{u}(\xi)|^{2} d \xi+\|u\|_{L^{2}}^{2}
\end{aligned}
$$

We note $I=\frac{1}{(2 \pi)^{3}} \int_{|\xi| \leq 1} \frac{1}{|\xi|^{4 s}}|\widehat{u}(\xi)|^{2} d \xi$. Using the fact that $\widehat{u}(0)=\int_{\mathbb{R}^{3}} u(x) d x=0$ and the Cauchy-Schwartz inequality on the interval $(0,1)$, we have

$$
\begin{aligned}
I & =\frac{1}{(2 \pi)^{3}} \int_{|\xi| \leq 1} \frac{1}{|\xi|^{4 s}}\left|\int_{0}^{1} \xi . \nabla \widehat{u}(\sigma \xi) d \sigma\right|^{2} d \xi \\
& \leq C \int_{|\xi| \leq 1} \frac{1}{|\xi|^{4 s-2}} \int_{0}^{1}|\nabla \widehat{u}(\sigma \xi)|^{2} d \sigma d \xi .
\end{aligned}
$$


Then, due to the fact that $\partial_{j} \widehat{u}(0)=i \int_{\mathbb{R}^{2}} x_{j} u(x) d x=0$, we get

$$
\begin{aligned}
I & \leq C \int_{|\xi| \leq 1} \frac{1}{|\xi|^{4 s-2}} \int_{0}^{1}\left(\sum_{i, j=1}^{3}\left|\int_{0}^{1} \xi_{j} \partial_{i} \partial_{j} \widehat{u}(r \sigma \xi) d r\right|^{2}\right) d \sigma d \xi \\
& \leq C \int_{|\xi| \leq 1} \frac{1}{|\xi|^{4 s-4}} \int_{0}^{1} \int_{0}^{1}\left|\nabla^{2} \widehat{u}(r \sigma \xi)\right|^{2} d r d \sigma d \xi .
\end{aligned}
$$

Finally, the continuous injection of $H^{2}\left(\mathbb{R}^{3}\right)$ into $L^{\infty}\left(\mathbb{R}^{3}\right)$ yields

$$
\begin{aligned}
I & \leq \frac{C}{7-4 s}\left\|\nabla^{2} \widehat{u}\right\|_{L^{\infty}}^{2} \\
& \leq \frac{C}{7-4 s}\left\|\nabla^{2} \widehat{u}\right\|_{H^{2}}^{2} \\
& \leq \frac{C}{7-4 s}\|u\|_{L^{2}(4)}^{2},
\end{aligned}
$$

and thus the inequality (4.7) is shown.

To get (4.8), using Fourier variables, we have

$$
\begin{aligned}
\left\|(-\Delta)^{-s} \nabla u\right\|_{L^{2}}^{2} & =\frac{1}{(2 \pi)^{3}} \int_{|\xi| \leq 1} \frac{1}{|\xi|^{4 s-2}}|\widehat{u}(\xi)|^{2} d \xi+\|u\|_{L^{2}}^{2} \\
& =\frac{1}{(2 \pi)^{3}} \int_{|\xi| \leq 1} \frac{1}{|\xi|^{4 s-2}}\left|\int_{0}^{1} \xi \cdot \nabla \widehat{u}(s \xi) d s\right|^{2} d \xi+\|u\|_{L^{2}}^{2} \\
& \leq \frac{1}{(2 \pi)^{3}} \int_{|\xi| \leq 1} \frac{1}{|\xi|^{4 s-4}}\left|\int_{0}^{1}\right| \nabla \widehat{u}(s \xi)|d s|^{2} d \xi+\|u\|_{L^{2}}^{2} .
\end{aligned}
$$

Using now Hölder inequalities, the fact that $4 s-4<3$ and the continuous injection of $H^{2}\left(\mathbb{R}^{3}\right)$ into $L^{\infty}\left(\mathbb{R}^{3}\right)$, we have

$$
\begin{aligned}
\left\|(-\Delta)^{-s} \nabla u\right\|_{L^{2}}^{2} & \leq C \int_{0}^{1} \int_{|\xi| \leq 1} \frac{1}{|\xi|^{4 s-4}}|\nabla \widehat{u}(s \xi)|^{2} d \xi d s+\|u\|_{L^{2}}^{2} \\
& \leq C\left(\int_{|\xi| \leq 1} \frac{1}{|\xi|^{4 s-4}} d \xi\right)\|\nabla \widehat{u}\|_{L^{\infty}}^{2}+\|u\|_{L^{2}}^{2} \\
& \leq \frac{C}{7-4 s}\|u\|_{L^{2}(3)}^{2}+\|u\|_{L^{2}}^{2} .
\end{aligned}
$$

In order to apply the lemma 4.2 to the non linear terms of the equation (4.4), we state the following lemma.

Lemma 4.3. Let $w \in \mathbb{H}^{2}(4)$ and $u$ obtained from $w$ via the Biot-Savart law (2.16). For all $C \in \mathbb{R}$, we have

$$
\int_{\mathbb{R}^{3}}(w(x)-C \Delta w(x)) \wedge u(x) d x=0 .
$$

Proof. In order to show this equality, we just have to look at the equality (2.9). An integration by parts gives directly (4.3). 
LEMMA 4.4. Let $w$ belongs to $H^{2}(4)$ and $s$ such that $0 \leq s<\frac{7}{4}$, then $u$ satisfies the equalities

$$
\begin{aligned}
& \text { (1) }\left((-\Delta)^{-s} \mathcal{L}(w),(-\Delta)^{-s} w\right)_{L^{2}}=-\left\|(-\Delta)^{\frac{1}{2}-s} w\right\|_{L^{2}}^{2}-\left(s-\frac{1}{4}\right)\left\|(-\Delta)^{-s} w\right\|_{L^{2}}^{2} . \\
& \text { (2) }\left((-\Delta)^{-s}\left(\frac{x}{2} \cdot \nabla \Delta w\right),(-\Delta)^{-s} w\right)_{L^{2}}=\left(s+\frac{5}{4}\right)\left\|(-\Delta)^{\frac{1}{2}-s} w\right\|_{L^{2}}^{2} .
\end{aligned}
$$

This lemma is easily obtained with a few integrations by parts, when passing into Fourier variables.

Let $V_{\infty}$ be the divergence free vector field obtained from $\Omega_{\infty}$ via the BiotSavart law (2.16) and $K$ the divergence free vector field obtained from $R$ via the Biot-Savart law. One defines the energy functional

$$
E_{0}(\tau)=\frac{1}{2}\left(\left\|(-\Delta)^{-\left(\frac{\theta}{2}+1\right)} R\right\|_{L^{2}}^{2}+\alpha e^{-\tau}\left\|(-\Delta)^{-\left(\frac{\theta+1}{2}\right)} R\right\|_{L^{2}}^{2}\right) .
$$

The next lemma establishes a $H^{-(\theta+2)}$-estimate which is necessary to obtain a good rate of convergence in Theorem 2.2 .

Lemma 4.5. Let $W \in C^{1}\left(\left(\tau_{0}, \tau_{\varepsilon}\right), \mathbb{H}^{1}(4)\right) \cap C^{0}\left(\left(\tau_{0}, \tau_{\varepsilon}\right), \mathbb{H}^{3}(4)\right)$ be the solution of (4.1). There exists a positive constant $\gamma_{0}$ such that, if $W_{\varepsilon}$ satisfies the condition (4.5) for some $\gamma$ such that $0<\gamma \leq \gamma_{0}$, then there exists a positive constant $C$ such that, for all $\tau \in\left[\tau_{0}, \tau_{\varepsilon}^{*}\right)$,

$$
\begin{aligned}
& \partial_{\tau} E_{0}+2 \theta E_{0}+\frac{1}{2}\left\|(-\Delta)^{-\left(\frac{\theta+1}{2}\right)} R\right\|_{L^{2}}^{2} \leq \\
& C M \gamma\left(\left\||X|^{4} R\right\|_{L^{2}}^{2}+\|\nabla R\|_{L^{2}}^{2}+\alpha^{2} e^{-2 \tau}\|\Delta R\|_{L^{2}(4)}^{2}\right) \\
& +C M^{2} \gamma\left(\frac{3}{2}-\theta\right)^{2} e^{-4 \tau} .
\end{aligned}
$$

Proof. To prove this lemma, we apply the operator $(-\Delta)^{-\left(\frac{\theta}{2}+1\right)}$ to $(4.4)$ and make the $L^{2}$-inner product of it with $(-\Delta)^{-\left(\frac{\theta}{2}+1\right)} R$. Applying Lemma 4.4 and through some easy computations, one has

$$
\begin{aligned}
& \frac{1}{2} \partial_{\tau}\left(\left\|(-\Delta)^{-\left(\frac{\theta}{2}+1\right)} R\right\|_{L^{2}}^{2}+\alpha e^{-\tau}\left\|(-\Delta)^{-\left(\frac{\theta+1}{2}\right)} R\right\|_{L^{2}}^{2}\right) \\
& +\varepsilon e^{-\tau}\left\|(-\Delta)^{-\frac{\theta}{2}} R\right\|_{L^{2}}^{2} \\
& +\left(\frac{\theta}{2}+\frac{3}{4}\right)\left\|(-\Delta)^{-\left(\frac{\theta}{2}+1\right)} R\right\|_{L^{2}}^{2}+ \\
& \left(1+\left(\frac{\theta}{2}+\frac{3}{4}\right) \alpha e^{-\tau}\right)\left\|(-\Delta)^{-\left(\frac{\theta+1}{2}\right)} R\right\|_{L^{2}}^{2}=I_{1}+I_{2},
\end{aligned}
$$

where

$$
\begin{aligned}
& I_{1}=\left((-\Delta)^{-\left(\frac{\theta}{2}+1\right)}\left(\operatorname{curl}\left(\left(W-\alpha e^{-\tau} \Delta W\right) \wedge U\right)\right),(-\Delta)^{-\left(\frac{\theta}{2}+1\right)} R\right)_{L^{2}}, \\
& I_{2}=e^{-2 \tau}\left((-\Delta)^{-\left(\frac{\theta}{2}+1\right)}\left(-\alpha \Delta \Omega_{\infty}-\varepsilon \Delta^{2} \Omega_{\infty}\right),(-\Delta)^{-\left(\frac{\theta}{2}+1\right)} R\right)_{L^{2}} .
\end{aligned}
$$

We start with the estimate of the easiest term, that is $I_{2}$. Through the Plancherel formula, one has 
ASYMPTOTIC PROFILES FOR THE SECOND GRADE FLUIDS EQUATIONS IN $\mathbb{R}^{3} \quad 143$

$$
I_{2}=e^{-2 \tau}\left((-\Delta)^{-\left(\frac{\theta}{2}+\frac{3}{2}\right)}\left(-\alpha \Delta \Omega_{\infty}-\varepsilon \Delta^{2} \Omega_{\infty}\right),(-\Delta)^{-\left(\frac{\theta+1}{2}\right)} R\right)_{L^{2}} .
$$

Using the Cauchy-Schwartz inequality, we get

$$
\begin{aligned}
I_{2} \leq \alpha e^{-2 \tau}\left\|(-\Delta)^{-\frac{\theta+1}{2}} \Omega_{\infty}\right\|_{L^{2}} & \left\|(-\Delta)^{-\left(\frac{\theta+1}{2}\right)} R\right\|_{L^{2}} \\
& +\varepsilon e^{-2 \tau}\left\|(-\Delta)^{-\frac{\theta-1}{2}} \Omega_{\infty}\right\|_{L^{2}}\left\|(-\Delta)^{-\left(\frac{\theta+1}{2}\right)} R\right\|_{L^{2}}
\end{aligned}
$$

Using the Lemma 4.2, the Young inequality and taking into account the good regularity of $\Omega_{\infty}$ and the inequality (4.6), one has

$$
\begin{aligned}
I_{2} & \leq C e^{-2 \tau}\left\|\Omega_{\infty}\right\|_{H^{2}(4)}\left\|(-\Delta)^{-\left(\frac{\theta+1}{2}\right)} R\right\|_{L^{2}} \\
& \leq \mu_{1}\left\|(-\Delta)^{-\left(\frac{\theta+1}{2}\right)} R\right\|_{L^{2}}^{2}+\frac{C|b|^{2}}{\mu_{1}} e^{-4 \tau} \\
& \leq \mu_{1}\left\|(-\Delta)^{-\left(\frac{\theta+1}{2}\right)} R\right\|_{L^{2}}^{2}+\frac{C M \gamma\left(\frac{3}{2}-\theta\right)^{2}}{\mu_{1}} e^{-4 \tau},
\end{aligned}
$$

where $\mu_{1}$ is a positive constant that will be made more precise later.

It remains to bound $I_{1}$. Using the Cauchy-Schwartz inequality and the lemmas 4.3 and 4.2 , we obtain

$$
\begin{aligned}
I_{1} & \leq C\left\|(-\Delta)^{-\left(\frac{\theta}{2}+1\right)} \nabla\left(\left(W-\alpha e^{-\tau} \Delta W\right) \wedge U\right)\right\|_{L^{2}}\left\|(-\Delta)^{-\left(\frac{\theta}{2}+1\right)} R\right\|_{L^{2}} \\
& \leq \frac{C}{\left(\frac{3}{2}-\theta\right)^{1 / 2}}\left\|\left(W-\alpha e^{-\tau} \Delta W\right) U\right\|_{L^{2}(4)}\left\|(-\Delta)^{-\left(\frac{\theta}{2}+1\right)} R\right\|_{L^{2}} \\
& \leq \frac{C}{\left(\frac{3}{2}-\theta\right)^{1 / 2}}\|U\|_{L^{\infty}}\left\|W-\alpha e^{-\tau} \Delta W\right\|_{L^{2}(4)}\left\|(-\Delta)^{-\left(\frac{\theta}{2}+1\right)} R\right\|_{L^{2}} .
\end{aligned}
$$

The inequality (2.18) of Lemma 2.4 with $p=2, q=6$ and $\eta=\frac{1}{2}$ and the continuous injection of $H^{1}\left(\mathbb{R}^{3}\right)$ into $L^{6}\left(\mathbb{R}^{3}\right)$ yield

$$
\begin{aligned}
I_{1} & \leq \frac{C}{\left(\frac{3}{2}-\theta\right)^{1 / 2}}\|W\|_{L^{2}}^{1 / 2}\|W\|_{L^{6}}^{1 / 2}\left\|W-\alpha e^{-\tau} \Delta W\right\|_{L^{2}(4)}\left\|(-\Delta)^{-\left(\frac{\theta}{2}+1\right)} R\right\|_{L^{2}} \\
& \leq \frac{C}{\left(\frac{3}{2}-\theta\right)^{1 / 2}}\|W\|_{H^{1}}\left(\|W\|_{L^{2}(4)}+\alpha e^{-\tau}\|\Delta W\|_{L^{2}(4)}\right)\left\|(-\Delta)^{-\left(\frac{\theta}{2}+1\right)} R\right\|_{L^{2}} \\
& \leq \mu_{2}\left\|(-\Delta)^{-\left(\frac{\theta}{2}+1\right)} R\right\|_{L^{2}}^{2} \\
& +\frac{C}{\mu_{2}\left(\frac{3}{2}-\theta\right)}\left(\|W\|_{L^{2}}^{2}+\|\nabla W\|_{L^{2}}^{2}\right)\left(\|W\|_{L^{2}(4)}^{2}+\alpha^{2} e^{-2 \tau}\|\Delta W\|_{L^{2}(4)}^{2}\right)
\end{aligned}
$$


where $\mu_{2}$ is a positive constant that will de made more precise later. Due to the decomposition (4.3), one has

$$
\begin{aligned}
I_{1} & \leq \mu_{2}\left\|(-\Delta)^{-\left(\frac{\theta}{2}+1\right)} R\right\|_{L^{2}}^{2} \\
& +\frac{C}{\mu_{2}\left(\frac{3}{2}-\theta\right)}\left(\|R\|_{L^{2}}^{2}+\|\nabla R\|_{L^{2}}^{2}\right)\left(\|W\|_{L^{2}(4)}^{2}+\alpha^{2} e^{-2 \tau}\|\Delta W\|_{L^{2}(4)}^{2}\right) \\
& +\frac{C e^{-2 \tau}}{\mu_{2}\left(\frac{3}{2}-\theta\right)}\left(\left\|\Omega_{\infty}\right\|_{L^{2}}^{2}+\left\|\nabla \Omega_{\infty}\right\|_{L^{2}}^{2}\right)\left(\|R\|_{L^{2}(4)}^{2}+\alpha^{2} e^{-2 \tau}\|\Delta R\|_{L^{2}(4)}^{2}\right) \\
& +\frac{C e^{-4 \tau}}{\mu_{2}\left(\frac{3}{2}-\theta\right)}\left(\left\|\Omega_{\infty}\right\|_{L^{2}}^{2}+\left\|\nabla \Omega_{\infty}\right\|_{L^{2}}^{2}\right)\left(\left\|\Omega_{\infty}\right\|_{L^{2}(4)}^{2}+\alpha^{2} e^{-2 \tau}\left\|\Delta \Omega_{\infty}\right\|_{L^{2}(4)}^{2}\right) .
\end{aligned}
$$

Finally, using the inequalities (4.5) and (4.6), we obtain $(4.13)$

$$
\begin{aligned}
I_{1} \leq \mu_{2}\left\|(-\Delta)^{-\left(\frac{\theta}{2}+1\right)} R\right\|_{L^{2}}^{2}+\frac{C M^{2} \gamma^{2}\left(\frac{3}{2}-\theta\right)^{3} e^{-4 \tau}}{\mu_{2}} & \\
& +\frac{C M \gamma\left(\frac{3}{2}-\theta\right)}{\mu_{2}}\left(\|R\|_{L^{2}(4)}^{2}+\|\nabla R\|_{L^{2}}^{2}+\alpha^{2} e^{-2 \tau}\|\Delta R\|_{L^{2}(4)}^{2}\right) .
\end{aligned}
$$

Combining (4.11), (4.12) and (4.13), it comes (4.14)

$$
\begin{aligned}
& \frac{1}{2} \partial_{\tau}\left(\left\|(-\Delta)^{-\left(\frac{\theta}{2}+1\right)} R\right\|_{L^{2}}^{2}+\alpha e^{-\tau}\left\|(-\Delta)^{-\left(\frac{\theta+1}{2}\right)} R\right\|_{L^{2}}^{2}\right)+\varepsilon e^{-\tau}\left\|(-\Delta)^{-\frac{\theta}{2}} R\right\|_{L^{2}}^{2} \\
& +\left(\theta+\frac{1}{2}\left(\frac{3}{2}-\theta-2 \mu_{2}\right)\right)\left\|(-\Delta)^{-\left(\frac{\theta}{2}+1\right)} R\right\|_{L^{2}}^{2} \\
& +\left(1-\mu_{1}+\left(\frac{\theta}{2}+\frac{3}{4}\right) \alpha e^{-\tau}\right)\left\|(-\Delta)^{-\left(\frac{\theta+1}{2}\right)} R\right\|_{L^{2}}^{2} \\
& \leq \frac{C M \gamma\left(\frac{3}{2}-\theta\right)}{\mu_{2}}\left(\|R\|_{L^{2}(4)}^{2}+\|\nabla R\|_{L^{2}}^{2}+\alpha^{2} e^{-2 \tau}\|\Delta R\|_{L^{2}(4)}^{2}\right)+ \\
& \frac{C M \gamma\left(\frac{3}{2}-\theta\right)^{2} e^{-4 \tau}}{\mu_{1}}+\frac{C M^{2} \gamma^{2}\left(\frac{3}{2}-\theta\right)^{3} e^{-4 \tau}}{\mu_{2}} .
\end{aligned}
$$

We set $\mu_{1}=\frac{1}{4}$ and $\mu_{2}=\frac{1}{2}\left(\frac{3}{2}-\theta\right)$. Recalling that $M \geq 2$ and $\gamma \leq 1$, we obtain

$$
\begin{aligned}
& \partial_{\tau} E_{0}+2 \theta E_{0}+\frac{3}{4}\left\|(-\Delta)^{-\left(\frac{\theta+1}{2}\right)} R\right\|_{L^{2}}^{2} \leq \\
& C M \gamma\left(\|R\|_{L^{2}(4)}^{2}+\|\nabla R\|_{L^{2}}^{2}+\alpha^{2} e^{-2 \tau}\|\Delta R\|_{L^{2}(4)}^{2}\right) \\
& +C M^{2} \gamma\left(\frac{3}{2}-\theta\right)^{2} e^{-4 \tau}
\end{aligned}
$$


Furthermore, using Fourier variables and Hölder inequalities, we see that

$$
\begin{aligned}
\|R\|_{L^{2}}^{2} & =\frac{1}{(2 \pi)^{3}} \int_{\mathbb{R}^{3}}|\widehat{R}(\xi)|^{2} d \xi \\
& \leq \frac{1}{(2 \pi)^{3}} \int_{\mathbb{R}^{3}}|\xi|^{\frac{2(1+\theta)}{2+\theta}}|\widehat{R}(\xi)|^{\frac{2(1+\theta)}{2+\theta}} \frac{1}{|\xi|^{\frac{2(1+\theta)}{2+\theta}}}|\widehat{R}(\xi)|^{\frac{2}{2+\theta}} d \xi \\
& \leq\left(\frac{1}{(2 \pi)^{3}} \int_{\mathbb{R}^{3}} \frac{1}{|\xi|^{2(\theta+1)}}|\widehat{R}(\xi)|^{2} d \xi\right)^{\frac{1+\theta}{2+\theta}} \\
& \left(\frac{1}{(2 \pi)^{3}} \int_{\mathbb{R}^{3}}|\xi|^{2}|\widehat{R}(\xi)|^{2} d \xi\right)^{\frac{1}{2+\theta}} \\
& \leq\left\|(-\Delta)^{-\left(\frac{\theta+1}{2}\right)} R\right\|_{L^{2}}^{\frac{2(1+\theta)}{2+\theta}}\|\nabla R\|_{L^{2}}^{\frac{2}{2+\theta}} .
\end{aligned}
$$

Using a convexity inequality, it is easy to get

$$
\|R\|_{L^{2}}^{2} \leq \frac{1}{\eta^{\frac{2+\theta}{1+\theta}}}\left(\frac{1+\theta}{2+\theta}\right)\left\|(-\Delta)^{-\left(\frac{\theta+1}{2}\right)} R\right\|_{L^{2}}^{2}+\frac{\eta^{2+\theta}}{2+\theta}\|\nabla R\|_{L^{2}}^{2},
$$

for all $0<\eta \leq 1$.

Via a short computation, using the fact that $1<\theta<\frac{3}{2}$ and $0<\eta \leq 1$, we obtain

$$
\|R\|_{L^{2}}^{2} \leq \frac{5}{7 \eta^{2}}\left\|(-\Delta)^{-\left(\frac{\theta+1}{2}\right)} R\right\|_{L^{2}}^{2}+\frac{\eta^{2}}{2}\|\nabla R\|_{L^{2}}^{2} .
$$

Applying (4.16) with $\eta=1$ and taking $\gamma$ small enough, the inequality (4.15) becomes

$$
\begin{aligned}
& \partial_{\tau} E_{0}+2 \theta E_{0}+\frac{1}{2}\left\|(-\Delta)^{-\left(\frac{\theta+1}{2}\right)} R\right\|_{L^{2}}^{2} \leq \\
& C M \gamma\left(\left\||X|^{4} R\right\|_{L^{2}}^{2}+\|\nabla R\|_{L^{2}}^{2}+\alpha^{2} e^{-2 \tau}\|\Delta R\|_{L^{2}(4)}^{2}\right)+C M^{2} \gamma\left(\frac{3}{2}-\theta\right)^{2} e^{-4 \tau}
\end{aligned}
$$

4.2. Estimates in $H^{1}\left(\mathbb{R}^{3}\right)$. This section is devoted to the $H^{1}$-estimate of the solutions of (4.4) under the condition (4.5). In particular, we see how the previous estimate in $\dot{H}^{-(1+\theta)}$ enables to absorb the terms involving the $L^{2}-$ norm of $R$. To obtain this $H^{1}$-estimate, we make the $L^{2}$-scalar product of (4.4) with $R$. We define the energy functional

$$
E_{1}(\tau)=\frac{1}{2}\left(\|R\|_{L^{2}}^{2}+\alpha e^{-\tau}\|\nabla R\|_{L^{2}}^{2}\right) .
$$

The estimate of $R$ in the Sobolev space $H^{1}\left(\mathbb{R}^{3}\right)$ is given by the next lemma.

LEMma 4.6. Let $W \in C^{1}\left(\left(\tau_{0}, \tau_{\varepsilon}\right), \mathbb{H}^{1}(4)\right) \cap C^{0}\left(\left(\tau_{0}, \tau_{\varepsilon}\right), \mathbb{H}^{3}(4)\right)$ be the solution of (4.1). There exist two positive constants $\gamma_{0}$ and $T_{0}$ such that, if $T \geq T_{0}$ and $W$ satisfies the condition (4.5) for some $\gamma$ such that $0<\gamma \leq \gamma_{0}$, then there exists a 
positive constant $C$ such that, for all $\tau \in\left[\tau_{0}, \tau_{\varepsilon}^{*}\right)$,

$$
\begin{aligned}
\partial_{\tau} E_{1}+3 E_{1}+\frac{1}{2}\|\nabla R\|_{L^{2}}^{2} \leq \frac{7}{4} \| & R \|_{L^{2}}^{2}+C M^{2} \gamma\left(\frac{3}{2}-\theta\right)^{2} e^{-4 \tau} \\
& +C M \gamma\left(\frac{3}{2}-\theta\right)^{2}\left(\|R\|_{L^{2}}^{2}+\alpha^{2} e^{-2 \tau}\|\Delta R\|_{L^{2}}^{2}\right) .
\end{aligned}
$$

Proof. We perform the $L^{2}$-scalar product of (4.4) with $R$. Through several integrations by parts, we obtain

$\frac{1}{2} \partial_{\tau}\left(\|R\|_{L^{2}}^{2}+\alpha e^{-\tau}\|\nabla R\|_{L^{2}}^{2}\right)+\varepsilon\|\Delta R\|_{L^{2}}^{2}+\left(1-\frac{\alpha}{4} e^{-\tau}\right)\|\nabla R\|_{L^{2}}^{2}-\frac{1}{4}\|R\|_{L^{2}}^{2}=I_{1}+I_{2}$,

where

$$
\begin{aligned}
& I_{1}=\left(\operatorname{curl}\left(\left(W-\alpha e^{-\tau} \Delta W\right) \wedge U\right), R\right)_{L^{2}}, \\
& I_{2}=e^{-2 \tau}\left(-\alpha \Delta \Omega_{\infty}-\varepsilon \Delta^{2} \Omega_{\infty}, R\right)_{L^{2}} .
\end{aligned}
$$

As usual, because of the good regularity of $\Omega_{\infty}$, the easiest term to estimate is $I_{2}$. Integrating by parts, one has

$$
I_{2}=e^{-2 \tau}\left(\alpha \nabla \Omega_{\infty}+\varepsilon \nabla \Delta \Omega_{\infty}, \nabla R\right)_{L^{2}} .
$$

Using the Hölder and Young inequalities and the inequality (4.6), we get

$$
\begin{aligned}
I_{2} & \leq e^{-2 \tau}\left(\alpha\left\|\nabla \Omega_{\infty}\right\|_{L^{2}}+\varepsilon\left\|\nabla \Delta \Omega_{\infty}\right\|_{L^{2}}\right)\|\nabla R\|_{L^{2}} \\
& \leq C|b|(\alpha+\varepsilon) e^{-2 \tau}\|\nabla R\|_{L^{2}} \\
& \leq \mu\|\nabla R\|_{L^{2}}^{2}+\frac{C M \gamma\left(\frac{3}{2}-\theta\right)^{2}}{\mu} e^{-4 \tau}
\end{aligned}
$$

where $\mu$ is a positive constant that will be made more precise later.

The last remaining term will be estimated by the same way, using the divergence free property of $U$. Integrating by parts, we obtain

$$
I_{1}=\left(\left(W-\alpha e^{-\tau} \Delta W\right) \wedge U, \operatorname{curl} R\right)_{L^{2}} .
$$

We recall that curl $K=R$ and curl $V_{\infty}=\Omega_{\infty}$ and we decompose $I_{1}$ as the sum of three terms

$$
I_{1}=I_{1}^{1}+I_{1}^{2}+I_{1}^{3}
$$

where

$$
\begin{aligned}
& I_{1}^{1}=\left(\left(W-\alpha e^{-\tau} \Delta W\right) \wedge K, \operatorname{curl} R\right)_{L^{2}}, \\
& I_{1}^{2}=e^{-\tau}\left(\left(R-\alpha e^{-\tau} \Delta R\right) \wedge V_{\infty}, \operatorname{curl} R\right)_{L^{2}}, \\
& I_{1}^{3}=e^{-2 \tau}\left(\left(\Omega_{\infty}-\alpha e^{-\tau} \Delta \Omega_{\infty}\right) \wedge V_{\infty}, \operatorname{curl} R\right)_{L^{2}} .
\end{aligned}
$$

The Hölder inequalities lead to

$$
\begin{aligned}
I_{1}^{1} & \leq C\left(\|K W\|_{L^{2}}+\alpha e^{-\tau}\|K \Delta W\|_{L^{2}}\right)\|\nabla R\|_{L^{2}} \\
& \leq C\|K\|_{L^{\infty}}\left(\|W\|_{L^{2}}+\alpha e^{-\tau}\|\Delta W\|_{L^{2}}\right)\|\nabla R\|_{L^{2}} .
\end{aligned}
$$


Applying the inequality (2.18) with $p=2, q=6$ and $\eta=\frac{1}{2}$ and using the continuous injection of $H^{1}\left(\mathbb{R}^{3}\right)$ into $L^{6}\left(\mathbb{R}^{3}\right)$, one gets

$$
\begin{aligned}
I_{1}^{1} & \leq C\|R\|_{L^{2}}^{1 / 2}\|R\|_{L^{6}}^{1 / 2}\left(\|W\|_{L^{2}}+\alpha e^{-\tau}\|\Delta W\|_{L^{2}}\right)\|\nabla R\|_{L^{2}} \\
& \leq C\|R\|_{L^{2}}^{1 / 2}\|R\|_{H^{1}}^{1 / 2}\left(\|W\|_{L^{2}}+\alpha e^{-\tau}\|\Delta W\|_{L^{2}}\right)\|\nabla R\|_{L^{2}} .
\end{aligned}
$$

Then, we use the Young inequality and the inequality (4.5). We obtain

$$
\begin{aligned}
I_{1}^{1} & \leq \mu\|\nabla R\|_{L^{2}}^{2}+\frac{C}{\mu}\left(\|W\|_{L^{2}}^{2}+\alpha^{2} e^{-2 \tau}\|\Delta W\|_{L^{2}}^{2}\right)\left(\|R\|_{L^{2}}^{2}+\|\nabla R\|_{L^{2}}^{2}\right) \\
& \leq \mu\|\nabla R\|_{L^{2}}^{2}+\frac{C M \gamma\left(\frac{3}{2}-\theta\right)^{2}}{\mu}\left(\|R\|_{L^{2}}^{2}+\|\nabla R\|_{L^{2}}^{2}\right) .
\end{aligned}
$$

The Hölder inequalities yield

$$
I_{1}^{2} \leq C e^{-\tau}\left\|V_{\infty}\right\|_{L^{\infty}}\left(\|R\|_{L^{2}}+\alpha e^{-\tau}\|\Delta R\|_{L^{2}}\right)\|\nabla R\|_{L^{2}} .
$$

Applying the inequality (2.18) of the lemma 2.4 with $p=2, q=6$ and $\eta=\frac{1}{2}$, and the inequality (4.6), we get

$$
\begin{aligned}
I_{1}^{2} & \leq C e^{-\tau}\left\|\Omega_{\infty}\right\|_{L^{2}}^{1 / 2}\left\|\Omega_{\infty}\right\|_{L^{6}}^{1 / 2}\left(\|R\|_{L^{2}}+\alpha e^{-\tau}\|\Delta R\|_{L^{2}}\right)\|\nabla R\|_{L^{2}} \\
& \leq C|b| e^{-\tau}\left(\|R\|_{L^{2}}+\alpha e^{-\tau}\|\Delta R\|_{L^{2}}\right)\|\nabla R\|_{L^{2}} \\
& \leq \mu\|\nabla R\|_{L^{2}}^{2}+\frac{C M \gamma\left(\frac{3}{2}-\theta\right)^{2}}{\mu}\left(\|R\|_{L^{2}}^{2}+\alpha^{2} e^{-2 \tau}\|\Delta R\|_{L^{2}}^{2}\right) .
\end{aligned}
$$

It remains to estimate $I_{1}^{3}$. By the same computations, we get

$$
\begin{aligned}
I_{1}^{3} & \leq \mu\|\nabla R\|_{L^{2}}^{2}+\frac{C}{\mu} e^{-4 \tau}\left\|V_{\infty}\right\|_{L^{\infty}}^{2}\left(\left\|\Omega_{\infty}\right\|_{L^{2}}^{2}+\alpha^{2} e^{-2 \tau}\left\|\Delta \Omega_{\infty}\right\|_{L^{2}}^{2}\right) \\
& \leq \mu\|\nabla R\|_{L^{2}}^{2}+\frac{C}{\mu} e^{-4 \tau}\left\|\Omega_{\infty}\right\|_{L^{2}}\left\|\Omega_{\infty}\right\|_{L^{6}}\left(\left\|\Omega_{\infty}\right\|_{L^{2}}^{2}+\alpha^{2} e^{-2 \tau}\left\|\Delta \Omega_{\infty}\right\|_{L^{2}}^{2}\right) \\
& \leq \mu\|\nabla R\|_{L^{2}}^{2}+\frac{C M^{2} \gamma^{2}\left(\frac{3}{2}-\theta\right)}{\mu} e^{-4 \tau} .
\end{aligned}
$$

In particular, we have shown that

$$
\begin{aligned}
I_{1} \leq 3 & \mu \nabla R \|_{L^{2}}^{2}+\frac{C M^{2} \gamma^{2}\left(\frac{3}{2}-\theta\right)^{4}}{\mu} e^{-4 \tau} \\
& +\frac{C M \gamma\left(\frac{3}{2}-\theta\right)^{2}}{\mu}\left(\|R\|_{L^{2}}^{2}+\|\nabla R\|_{L^{2}}^{2}+\alpha^{2} e^{-2 \tau}\|\Delta R\|_{L^{2}}^{2}\right) .
\end{aligned}
$$

Thus, due to the inequalities (4.20) and (4.21), the inequality (4.19) becomes

$$
\begin{aligned}
& \partial_{\tau} E_{1}+3 E_{1}+\left(1-4 \mu-\frac{7 \alpha}{4} e^{-\tau}\right)\|\nabla R\|_{L^{2}}^{2} \leq \frac{7}{4}\|R\|_{L^{2}}^{2}+\frac{C M^{2} \gamma\left(\frac{3}{2}-\theta\right)^{2}}{\mu} e^{-4 \tau} \\
& +\frac{C M \gamma\left(\frac{3}{2}-\theta\right)^{2}}{\mu}\left(\|R\|_{L^{2}}^{2}+\|\nabla R\|_{L^{2}}^{2}+\alpha^{2} e^{-2 \tau}\|\Delta R\|_{L^{2}}^{2}\right) .
\end{aligned}
$$


Taking $\gamma_{0}$ and $\mu$ small enough and $T=e^{\tau_{0}}$ large enough, we obtain the inequality $(4.23)$

$$
\begin{aligned}
& \partial_{\tau} E_{1}+3 E_{1}+\frac{1}{2}\|\nabla R\|_{L^{2}}^{2} \leq \frac{7}{4}\|R\|_{L^{2}}^{2}+C M \gamma\left(\frac{3}{2}-\theta\right)^{2}\left(\|R\|_{L^{2}}^{2}+\alpha^{2} e^{-2 \tau}\|\Delta R\|_{L^{2}}^{2}\right) \\
& +C M^{2} \gamma\left(\frac{3}{2}-\theta\right)^{2} e^{-4 \tau},
\end{aligned}
$$

that concludes the proof of this lemma.

In order to achieve the $H^{1}$-estimate of $R$, we now combine the energy inequalities (4.10) and (4.18). Using the interpolation inequality (4.16), we get, from the inequality (4.18),

$$
\begin{aligned}
& \partial_{\tau} E_{1}+3 E_{1}+\frac{1}{2}\|\nabla R\|_{L^{2}}^{2} \leq \frac{7}{4}\left(\frac{5}{7 \eta^{2}}\left\|(-\Delta)^{-\left(\frac{1+\theta}{2}\right)} R\right\|_{L^{2}}^{2}+\frac{\eta^{2}}{2}\|\nabla R\|_{L^{2}}^{2}\right) \\
& +C M \gamma\left(\frac{3}{2}-\theta\right)^{2}\left(\left\|(-\Delta)^{-\left(\frac{1+\theta}{2}\right)} R\right\|_{L^{2}}^{2}+\|\nabla R\|_{L^{2}}^{2}+\alpha^{2} e^{-2 \tau}\|\Delta R\|_{L^{2}}^{2}\right) \\
& +C M^{2} \gamma\left(\frac{3}{2}-\theta\right)^{2} e^{-4 \tau},
\end{aligned}
$$

where $0<\eta \leq 1$.

Taking $\eta=\sqrt{\frac{2}{7}}$ and $\gamma$ sufficiently small, we get

$$
\begin{aligned}
& \partial_{\tau} E_{1}+3 E_{1}+\frac{1}{4}\|\nabla R\|_{L^{2}}^{2} \leq\left(\frac{35}{8}+C M \gamma\left(\frac{3}{2}-\theta\right)^{2}\right)\left\|(-\Delta)^{-\left(\frac{1+\theta}{2}\right)} R\right\|_{L^{2}}^{2} \\
& +C M \gamma\left(\frac{3}{2}-\theta\right)^{2}\left(\left\|(-\Delta)^{-\left(\frac{1+\theta}{2}\right)} R\right\|_{L^{2}}^{2}+\alpha^{2} e^{-2 \tau}\|\Delta R\|_{L^{2}}^{2}\right)+C M^{2} \gamma\left(\frac{3}{2}-\theta\right)^{2} e^{-4 \tau} .
\end{aligned}
$$

Using the two energies $E_{0}$ and $E_{1}$, we define

$$
E_{2}=6 E_{0}+E_{1} \text {. }
$$

Combining the inequalities (4.10) and (4.25) and setting $\gamma$ sufficiently small, it is easy to check that

$$
\begin{aligned}
& \partial E_{2}(\tau)+2 \theta E_{2}(\tau)+\left\|(-\Delta)^{-\left(\frac{1+\theta}{2}\right)} R\right\|_{L^{2}}^{2}+\frac{1}{4}\|\nabla R\|_{L^{2}}^{2} \leq \\
& C M \gamma\left(\left\||X|^{4} R\right\|_{L^{2}}^{2}+\alpha^{2} e^{-2 \tau}\|\Delta R\|_{L^{2}}^{2}\right)^{2}+C M^{2} \gamma\left(\frac{3}{2}-\theta\right)^{2} e^{-4 \tau}
\end{aligned}
$$

4.3. Estimates in $H^{2}\left(\mathbb{R}^{3}\right)$. We now perform a $H^{2}$-estimate for the solution $R$ of (4.4) under the smallness assumption (4.5). To this end, we consider the $L^{2}$-scalar product of (4.4) with $-\Delta R$. We define the functional

$$
E_{3}(\tau)=\frac{1}{2}\left(\|\nabla R\|_{L^{2}}^{2}+\alpha e^{-\tau}\|\Delta R\|_{L^{2}}^{2}\right) .
$$

The next lemma gives the estimate of $R$ in the space $H^{2}\left(\mathbb{R}^{3}\right)$.

Lemma 4.7. Let $W \in C^{1}\left(\left(\tau_{0}, \tau_{\varepsilon}\right), \mathbb{H}^{1}(4)\right) \cap C^{0}\left(\left(\tau_{0}, \tau_{\varepsilon}\right), \mathbb{H}^{3}(4)\right)$ be the solution of (4.1). There exist two positive constants $\gamma_{0}$ and $T_{0}$ such that, if $T \geq T_{0}$ and $W$ satisfies the condition (4.5) for some positive constant $\gamma$ such that $\gamma \leq \gamma_{0}$, then 
there exists $C>0$ such that, for all $\tau \in\left[\tau_{0}, \tau_{\varepsilon}^{*}\right)$,

$$
\begin{aligned}
& \partial_{\tau} E_{3}+3 E_{3}+\frac{1}{2}\|\Delta R\|_{L^{2}}^{2} \leq \frac{9}{4}\|\nabla R\|_{L^{2}}^{2}+C M \gamma\left(\frac{3}{2}-\theta\right)^{2}\left(\|R\|_{L^{2}}^{2}+\|\nabla R\|_{L^{2}}^{2}\right) \\
& +C M^{2} \gamma\left(\frac{3}{2}-\theta\right)^{2} e^{-\frac{7 \tau}{2}} .
\end{aligned}
$$

Proof. The proof of Lemma 4.7 is made through the $L^{2}$-scalar product of (4.4) with $-\Delta R$. First of all, we remark that

$$
\operatorname{curl}\left(\left(W-\alpha e^{-\tau} \Delta W\right) \wedge U\right)=U . \nabla\left(W-\alpha e^{-\tau} \Delta W\right)-\left(W-\alpha e^{-\tau} \Delta W\right) . \nabla U .
$$

Making some computations that we let to the reader involving integrations by parts and the divergence free property of $U$, we obtain

$\partial_{\tau}\left(\|\nabla R\|_{L^{2}}^{2}+\alpha e^{-\tau}\|\Delta R\|_{L^{2}}^{2}\right)+\left(1-\frac{3 \alpha}{4} e^{-\tau}\right)\|\Delta R\|_{L^{2}}^{2}=\frac{3}{4}\|\nabla R\|_{L^{2}}^{2}+I_{1}+I_{2}+I_{3}$,

where

$$
\begin{aligned}
& I_{1}=\left(-U \cdot \nabla\left(W-\alpha e^{-\tau} \Delta W\right), \Delta R\right)_{L^{2}}, \\
& I_{2}=\left(\left(W-\alpha e^{-\tau} \Delta W\right) \cdot \nabla U, \Delta R\right)_{L^{2}}, \\
& I_{3}=e^{-2 \tau}\left(\alpha \Delta \Omega_{\infty}+\varepsilon \Delta^{2} \Omega_{\infty}, \Delta R\right)_{L^{2}} .
\end{aligned}
$$

Like in the previous estimates, the easiest term is $I_{3}$. Indeed, using Hölder and Young inequalities and the inequality (4.6), one has

$$
\begin{aligned}
I_{3} & \leq e^{-2 \tau}\left(\alpha\left\|\Delta \Omega_{\infty}\right\|_{L^{2}}+\varepsilon\left\|\Delta^{2} \Omega_{\infty}\right\|_{L^{2}}\right)\|\Delta R\|_{L^{2}} \\
& \leq \mu\|\Delta R\|^{2}+\frac{C M \gamma\left(\frac{3}{2}-\theta\right)^{2}}{\mu} e^{-4 \tau},
\end{aligned}
$$

where $\mu$ is a positive constant which will be made more precise later.

We now look for an estimate of $I_{1}$. We decompose it as follows:

$$
I_{1}=I_{1}^{1}+I_{1}^{2}+I_{1}^{3}
$$

where

$$
\begin{aligned}
& I_{1}^{1}=-e^{-\tau}\left(K . \nabla\left(\Omega_{\infty}-\alpha e^{-\tau} \Delta \Omega_{\infty}\right), \Delta R\right)_{L^{2}}, \\
& I_{1}^{2}=-e^{-2 \tau}\left(V_{\infty} \cdot \nabla\left(\Omega_{\infty}-\alpha e^{-\tau} \Delta \Omega_{\infty}\right), \Delta R\right)_{L^{2}}, \\
& I_{1}^{3}=-\left(U \cdot \nabla\left(R-\alpha e^{-\tau} \Delta R\right), \Delta R\right)_{L^{2}} .
\end{aligned}
$$

Due to the smoothness of $\Omega_{\infty}$ and the inequality (2.18), we get

$$
\begin{aligned}
I_{1}^{1} & \leq e^{-\tau}\|K\|_{L^{\infty}}\left(\left\|\nabla \Omega_{\infty}\right\|_{L^{2}}+\alpha e^{-\tau}\left\|\nabla \Delta \Omega_{\infty}\right\|_{L^{2}}\right)\|\Delta R\|_{L^{2}} \\
& \leq C|b| e^{-\tau}\|R\|_{L^{2}}^{1 / 2}\|R\|_{L^{6}}^{1 / 2}\|\Delta R\|_{L^{2}} .
\end{aligned}
$$


The continuous injection of $H^{1}\left(\mathbb{R}^{3}\right)$ into $L^{6}\left(\mathbb{R}^{3}\right)$, Young inequality and the inequality (4.6) yield

$$
\begin{aligned}
I_{1}^{1} & \leq C|b| e^{-\tau}\|R\|_{H^{1}}\|\Delta R\|_{L^{2}} \\
& \leq \mu\|\Delta R\|_{L^{2}}^{2}+\frac{C M \gamma\left(\frac{3}{2}-\theta\right)^{2}}{\mu} e^{-2 \tau}\left(\|R\|_{L^{2}}^{2}+\|\nabla R\|_{L^{2}}^{2}\right) .
\end{aligned}
$$

Doing the same computations, we get

$$
I_{1}^{2} \leq \mu\|\Delta R\|_{L^{2}}^{2}+\frac{C M^{2} \gamma^{2}\left(\frac{3}{2}-\theta\right)^{4}}{\mu} e^{-4 \tau} .
$$

The divergence free property of $U$ and an integration by parts imply

$$
I_{1}^{3}=(U \cdot \nabla R, \Delta R)_{L^{2}} .
$$

Thus, using the Hölder and Young inequalities, Lemma 2.4 and the inequality (4.5), we obtain

$$
\begin{aligned}
I_{1}^{3} & \leq\|U\|_{L^{\infty}}\|\nabla R\|_{L^{2}}\|\Delta R\|_{L^{2}} \\
& \leq C\|W\|_{L^{2}}^{1 / 2}\|W\|_{L^{6}}^{1 / 2}\|\nabla R\|_{L^{2}}\|\Delta R\|_{L^{2}} \\
& \leq C\|W\|_{H^{1}}\|\nabla R\|_{L^{2}}\|\Delta R\|_{L^{2}} \\
& \leq \mu\|\Delta R\|_{L^{2}}^{2}+\frac{C M \gamma\left(\frac{3}{2}-\theta\right)^{2}}{\mu}\|\nabla R\|_{L^{2}}^{2} .
\end{aligned}
$$

Consequently, we have shown that

$$
I_{1} \leq 3 \mu\|\Delta R\|_{L^{2}}^{2}+\frac{C M \gamma\left(\frac{3}{2}-\theta\right)^{2}}{\mu}\left(\|R\|_{L^{2}}^{2}+\|\nabla R\|_{L^{2}}^{2}\right)+\frac{C M^{2} \gamma^{2}\left(\frac{3}{2}-\theta\right)^{4}}{\mu} e^{-4 \tau} .
$$

It remains to estimate $I_{2}$. We set

$$
I_{2}=I_{2}^{1}+I_{2}^{2}
$$

where

$$
\begin{aligned}
& I_{2}^{1}=-(W \cdot \nabla U, \Delta R)_{L^{2}}, \\
& I_{2}^{2}=\alpha e^{-\tau}(\Delta W \cdot \nabla U, \Delta R)_{L^{2}}
\end{aligned}
$$

Recalling that $W=e^{-\tau} \Omega_{\infty}+R$ and using Hölder and Young inequalities and the inequality (2.19) with $p=4$, one has

$$
\begin{aligned}
I_{2}^{1} & \leq\|W\|_{L^{4}}\|\nabla U\|_{L^{4}}\|\Delta R\|_{L^{2}} \\
& \leq C\|W\|_{L^{4}}^{2}\|\Delta R\|_{L^{2}} \\
& \leq \mu\|\Delta R\|_{L^{2}}^{2}+\frac{C}{\mu}\|W\|_{L^{4}}^{4} \\
& \leq \mu\|\Delta R\|_{L^{2}}^{2}+\frac{C}{\mu}\left(e^{-4 \tau}\left\|\Omega_{\infty}\right\|_{L^{4}}^{4}+\|R\|_{L^{4}}^{4}\right) .
\end{aligned}
$$


The condition (4.6) and the continuous injection of $H^{1}\left(\mathbb{R}^{3}\right)$ into $L^{4}\left(\mathbb{R}^{3}\right)$ yield

$$
\begin{aligned}
I_{2}^{1} & \leq \mu\|\Delta R\|_{L^{2}}^{2}+\frac{C M^{2} \gamma^{2}\left(\frac{3}{2}-\theta\right)^{4}}{\mu} e^{-4 \tau}+\frac{C}{\mu}\|R\|_{H^{1}}^{4} \\
& \leq \mu\|\Delta R\|_{L^{2}}^{2}+\frac{C M^{2} \gamma^{2}\left(\frac{3}{2}-\theta\right)^{4}}{\mu} e^{-4 \tau}+\frac{C M \gamma\left(\frac{3}{2}-\theta\right)^{2}}{\mu}\left(\|R\|_{L^{2}}^{2}+\|\nabla R\|_{L^{2}}^{2}\right) .
\end{aligned}
$$

Using the inequality (2.18) with $p=2, q=6$ and $\eta=\frac{1}{2}$ and the continuous injection of $H^{1}\left(\mathbb{R}^{3}\right)$ into $L^{6}\left(\mathbb{R}^{3}\right)$, we obtain

$$
\begin{aligned}
I_{2}^{2} & \leq \alpha e^{-\tau}\left(\|\Delta R\|_{L^{2}}+e^{-\tau}\left\|\Delta \Omega_{\infty}\right\|_{L^{2}}\right)\|\nabla U\|_{L^{\infty}}\|\Delta R\|_{L^{2}} \\
& \leq C \alpha e^{-\tau}\left(\|\Delta R\|_{L^{2}}+e^{-\tau}\left\|\Delta \Omega_{\infty}\right\|_{L^{2}}\right)\|\nabla W\|_{L^{2}}^{1 / 2}\|\nabla W\|_{L^{6}}^{1 / 2}\|\Delta R\|_{L^{2}} \\
& \leq C \alpha e^{-\tau}\left(\|\Delta R\|_{L^{2}}+e^{-\tau}\left\|\Delta \Omega_{\infty}\right\|_{L^{2}}\right)\|\nabla W\|_{L^{2}}^{1 / 2}\|W\|_{H^{2}}^{1 / 2}\|\Delta R\|_{L^{2}} .
\end{aligned}
$$

We set $\delta=M \gamma\left(\frac{3}{2}-\theta\right)^{2}$. Taking into account the inequalities (4.6) and (4.5), it comes,

$$
\begin{aligned}
I_{2}^{2} & \leq C \delta^{1 / 2} e^{-\frac{3 \tau}{4}}\left(\|\Delta R\|_{L^{2}}+\delta^{1 / 2} e^{-\tau}\right)\|\Delta R\|_{L^{2}} \\
& \leq C \delta^{1 / 2} e^{-\frac{3 \tau}{4}}\|\Delta R\|_{L^{2}}^{2}+C \delta e^{-\frac{7 \tau}{4}}\|\Delta R\|_{L^{2}} \\
& \leq C\left(\delta^{1 / 2} e^{-\frac{3 \tau}{4}}+\delta\right)\|\Delta R\|_{L^{2}}^{2}+C \delta e^{-\frac{7 \tau}{2}} \\
& \leq C M \gamma^{1 / 2}\left(\frac{3}{2}-\theta\right)\|\Delta R\|_{L^{2}}^{2}+C M \gamma\left(\frac{3}{2}-\theta\right)^{2} e^{-\frac{7 \tau}{2}} .
\end{aligned}
$$

Finally, we have shown,

$$
\begin{aligned}
& I_{2} \leq\left(C M \gamma^{1 / 2}\left(\frac{3}{2}-\theta\right)+\mu\right)\|\Delta R\|_{L^{2}}^{2}+\frac{C M \gamma\left(\frac{3}{2}-\theta\right)^{2}}{\mu}\left(\|R\|_{L^{2}}^{2}+\|\nabla R\|_{L^{2}}^{2}\right) \\
& +\frac{C M^{2} \gamma\left(\frac{3}{2}-\theta\right)^{2}}{\mu} e^{-\frac{7 \tau}{2}} .
\end{aligned}
$$

Going back to (4.28), the inequalities (4.29), (4.30) and (4.31) imply

$$
\begin{aligned}
& \partial_{\tau} E_{3}+3 E_{3}+\left(1-5 \mu-\frac{9 \alpha}{4} e^{-\tau}\right)\|\Delta R\|_{L^{2}}^{2} \\
& \leq \frac{9}{4}\|\nabla R\|_{L^{2}}^{2}+C M \gamma^{1 / 2}\left(\frac{3}{2}-\theta\right)\|\Delta R\|_{L^{2}}^{2} \\
& +\frac{C M \gamma\left(\frac{3}{2}-\theta\right)^{2}}{\mu}\left(\|R\|_{L^{2}}^{2}+\|\nabla R\|_{L^{2}}^{2}\right)+\frac{C M^{2} \gamma\left(\frac{3}{2}-\theta\right)^{2}}{\mu} e^{-\frac{7 \tau}{2}} .
\end{aligned}
$$

We take $\gamma_{0}$ and $\mu$ small enough and $T=e^{\tau_{0}}$ large enough compared to $\alpha$ and obtain (4.33)

$$
\begin{aligned}
& \partial_{\tau} E_{3}+3 E_{3}+\frac{1}{2}\|\Delta R\|_{L^{2}}^{2} \leq \frac{9}{4}\|\nabla R\|_{L^{2}}^{2}+C M \gamma\left(\frac{3}{2}-\theta\right)^{2}\left(\|R\|_{L^{2}}^{2}+\|\nabla R\|_{L^{2}}^{2}\right) \\
& +C M^{2} \gamma\left(\frac{3}{2}-\theta\right)^{2} e^{-\frac{7 \tau}{2}} .
\end{aligned}
$$

To achieve the $H^{2}$-estimate, we combine $E_{2}$ and $E_{3}$ to define the functional

$$
E_{4}=12 E_{2}+E_{3} \text {. }
$$


Taking into account the two inequalities (4.26) and (4.27), we see that $E_{4}$ satisfies

$$
\begin{aligned}
& \partial_{\tau} E_{4}+2 \theta E_{4}+12\left\|(-\Delta)^{-\left(\theta-\frac{1}{4}\right)} R\right\|_{L^{2}}^{2} \\
& +\frac{3}{4}\|\nabla R\|_{L^{2}}^{2}+\frac{1}{2}\|\Delta R\|_{L^{2}}^{2} \leq \\
& +C M \gamma\left(\|R\|_{L^{2}}^{2}+\|\nabla R\|_{L^{2}}^{2}+\left\||X|^{4} R\right\|_{L^{2}}^{2}+\alpha^{2} e^{-2 \tau}\|\Delta R\|_{L^{2}}^{2}\right) \\
& +C M^{2} \gamma\left(\frac{3}{2}-\theta\right)^{2} e^{-\frac{7 \tau}{2}} .
\end{aligned}
$$

Using again the interpolation inequality (4.16) and taking $\gamma_{0}$ small enough, this inequality becomes

$$
\begin{aligned}
& \partial_{\tau} E_{4}+2 \theta E_{4}+10\left\|(-\Delta)^{-\left(\theta-\frac{1}{4}\right)} R\right\|_{L_{2}^{2}}^{2}+\frac{1}{2}\|\nabla R\|_{L^{2}}^{2}+\frac{1}{4}\|\Delta R\|_{L^{2}}^{2} \leq \\
& C M \gamma\left\||X|^{4} R\right\|_{L^{2}}^{2}+C M^{2} \gamma\left(\frac{3}{2}-\theta\right)^{2} e^{-\frac{7 \tau}{2}} .
\end{aligned}
$$

4.4. Estimates in $H^{2}(4)$. To finish the energy estimates, we have to work in weighted spaces. In order to perform estimates in weighted Lebesgue norms, and additionally absorb the term involving the weighted norm of $R$ in the right hand side of the inequality (4.35), we make the $L^{2}$-inner product of (4.4) with $|X|^{8}\left(R-\alpha e^{-\tau} \Delta R\right)$. One defines the energy functional

$$
E_{5}=\frac{1}{2}\left\||X|^{4}\left(R-\alpha e^{-\tau} \Delta R\right)\right\|_{L^{2}}^{2} .
$$

The next lemma summarizes the terms provided by the linear part of (4.4), when making the $L^{2}$-scalar product with $|X|^{8}\left(R-\alpha e^{-\tau} \Delta R\right)$.

LEMMA 4.8. Let $u$ be a divergence free vector field of $\mathbb{H}^{3}(4), a \in \mathbb{R}$ and $F(u)=$ $|x|^{8}(u-a \Delta u)$. The five next equalities hold.

$$
\begin{gathered}
(\Delta u, F(u))_{L^{2}}=36\left\||x|^{3} u\right\|_{L^{2}}^{2}-\left\||x|^{4} \nabla u\right\|_{L^{2}}^{2}-a\left\||x|^{4} \Delta u\right\|_{L^{2}}^{2} \cdot \\
\left(\frac{x}{2} \cdot \nabla u, F(u)\right)_{L^{2}}=-\frac{11}{4}\left\||x|^{4} u\right\|_{L^{2}}^{2}-\frac{9 a}{4}\left\||x|^{4} \nabla u\right\|_{L^{2}}^{2}+4 a\left\||x|^{3}(x . \nabla u)\right\|_{L^{2}}^{2}
\end{gathered}
$$

$$
\begin{aligned}
& (\mathcal{L}(u), F(u))_{L^{2}}=-\frac{7}{4}\left\||x|^{4} u\right\|_{L^{2}}^{2}-\left(1+\frac{5 a}{4}\right)\left\||x|^{4} \nabla u\right\|_{L^{2}}^{2}-a\left\||x|^{4} \Delta u\right\|_{L^{2}}^{2} \\
& +4 a\left\||x|^{3}(x . \nabla u)\right\|_{L^{2}}^{2}+36(1-a)\left\||x|^{3} u\right\|_{L^{2}}^{2} .
\end{aligned}
$$

$$
\begin{aligned}
\left(\Delta^{2} u, F(u)\right)_{L^{2}}= & \left\|\left.|| x\right|^{4} \Delta u\right\|_{L^{2}}^{2}-16\left\||x|^{3} \nabla u\right\|_{L^{2}}^{2}-96\left\||x|^{2}(x . \nabla u)\right\|_{L^{2}}^{2} \\
& +1512\left\||x|^{2} u\right\|_{L^{2}}^{2}+a\left\||x|^{4} \nabla \Delta u\right\|_{L^{2}}^{2}-36 a\left\||x|^{3} \Delta u\right\|_{L^{2}}^{2}
\end{aligned}
$$

$$
\begin{aligned}
\left(\frac{x}{2} \cdot \nabla \Delta u, F(u)\right)_{L^{2}}=\frac{13}{4}\left\||x|^{4} \nabla u\right\|_{L^{2}}^{2} & +\frac{11 a}{4}\left\||x|^{4} \Delta u\right\|_{L^{2}}^{2} \\
& +4\left\||x|^{3}(x \cdot \nabla u)\right\|_{L^{2}}^{2}-180\left\||x|^{3} u\right\|_{L^{2}}^{2}
\end{aligned}
$$


There is no difficulty in the proof of this lemma, which is let to the reader. It is only a consequence of many integrations by parts.

The next lemma enables us to achieve the $\mathbb{H}^{2}(4)$-estimate of $R$.

LEMma 4.9. Let $W \in C^{1}\left(\left(\tau_{0}, \tau_{\varepsilon}\right), \mathbb{H}^{1}(4)\right) \cap C^{0}\left(\left(\tau_{0}, \tau_{\varepsilon}\right), \mathbb{H}^{3}(4)\right)$ be the solution of (4.1). There exist two positive constants $\gamma_{0}$ and $T_{0}$ such that, if $T \geq T_{0}$ and $W$ satisfy the condition (4.5) for some positive constant such that $\gamma \leq \gamma_{0}$, then there exists $C>0$ such that, for all $\tau \in\left[\tau_{0}, \tau_{\varepsilon}^{*}\right)$,

$$
\begin{aligned}
& \partial_{\tau} E_{5}+3 E_{5}+\frac{1}{16}\left\||X|^{4} R\right\|_{L^{2}}^{2}+ \\
& \left(\frac{\alpha}{2} e^{-\tau}+\frac{\alpha^{2}}{4} e^{-2 \tau}\right)\left\||X|^{4} \Delta R\right\|_{L^{2}}^{2} \leq K_{1}\|R\|_{L^{2}}^{2} \\
& +C M \gamma^{1 / 4}\left(\frac{3}{2}-\theta\right)^{1 / 2}\left(\|R\|_{L^{2}}^{2}+\|\nabla R\|_{L^{2}}^{2}+\|\Delta R\|_{L^{2}}^{2}\right) \\
& +C M^{2} \gamma\left(\frac{3}{2}-\theta\right)^{2} e^{-4 \tau}
\end{aligned}
$$

where $K_{1}$ is a positive constant independent of the parameters.

Proof. To obtain the inequality (4.41) of this lemma, we perform the $L^{2}$-inner product of (4.4) with $|X|^{8}\left(R-\alpha e^{-\tau} \Delta R\right)$. We deliberately omit the positive terms obtained from $\varepsilon \Delta^{2} W$ which do not play any role in the next estimates. Using Lemma (4.8) and making some easy computations, one obtains

$$
\begin{aligned}
& \frac{1}{2} \partial_{\tau}\left(\left\||X|^{4}\left(R-\alpha e^{-\tau} \Delta R\right)\right\|_{L^{2}}^{2}\right)+\frac{7}{4}\left\||X|^{4} R\right\|_{L^{2}}^{2}+\left(1+\frac{7 \alpha}{2} e^{-\tau}\right)\left\||X|^{4} \nabla R\right\|_{L^{2}}^{2} \\
& +\left(\alpha e^{-\tau}+\frac{7 \alpha^{2}}{4} e^{-2 \tau}\right)\left\||X|^{4} \Delta R\right\|_{L^{2}}^{2}-108 \alpha e^{-\tau}\left\||X|^{3} R\right\|_{L^{2}}^{2}= \\
& 36\left\||X|^{3} R\right\|_{L^{2}}^{2}+I_{1}+I_{2}+I_{3}+I_{4},
\end{aligned}
$$

where

$$
\begin{aligned}
& I_{1}=\left(-U \cdot \nabla\left(W-\alpha e^{-\tau} \Delta W\right),|X|^{8}\left(R-\alpha e^{-\tau} \Delta R\right)\right)_{L^{2}}, \\
& I_{2}=\left(\left(W-\alpha e^{-\tau} \Delta W\right) \cdot \nabla U,|X|^{8}\left(R-\alpha e^{-\tau} \Delta R\right)\right)_{L^{2}}, \\
& I_{3}=\left(-\varepsilon e^{-2 \tau} \Delta^{2} \Omega_{\infty}-\alpha e^{-2 \tau} \Delta \Omega_{\infty},|X|^{8}\left(R-\alpha e^{-\tau} \Delta R\right)\right)_{L^{2}}, \\
& I_{4}=\varepsilon e^{-\tau}\left(16\left\||X|^{3} \nabla R\right\|_{L^{2}}^{2}+96\left\||X|^{2}(X . \nabla R)\right\|_{L^{2}}^{2}+36 \alpha e^{-\tau}\left\||X|^{3} \Delta R\right\|_{L^{2}}^{2}\right) .
\end{aligned}
$$

In the proof of this lemma, we use the notation

$$
\delta=M \gamma\left(\frac{3}{2}-\theta\right)^{2}
$$


As usual, $I_{3}$ is the easiest term to estimate. Indeed, due to the smoothness of $\Omega_{\infty}$ and the inequality (4.6), we get

$$
\begin{aligned}
I_{3} & \leq C e^{-2 \tau}\left\||X|^{4}\left(\alpha \Delta \Omega_{\infty}+\varepsilon \Delta^{2} \Omega_{\infty}\right)\right\|_{L^{2}}\left(\left\||X|^{4} R\right\|_{L^{2}}+\alpha e^{-\tau}\left\||X|^{4} \Delta R\right\|_{L^{2}}\right) \\
& \leq \mu\left\||X|^{4} R\right\|_{L^{2}}^{2}+\mu \alpha^{2} e^{-2 \tau}\left\||X|^{4} \Delta R\right\|_{L^{2}}^{2}+\frac{C|b|^{2}}{\mu} e^{-4 \tau} \\
& \leq \mu\left\||X|^{4} R\right\|_{L^{2}}^{2}+\mu \alpha^{2} e^{-2 \tau}\left\||X|^{4} \Delta R\right\|_{L^{2}}^{2}+\frac{C M \gamma\left(\frac{3}{2}-\theta\right)^{2}}{\mu} e^{-4 \tau},
\end{aligned}
$$

where $\mu$ is a positive constant that will be made more precise later.

We now give an estimate of $I_{4}$, which is also quite simple to bound. We just need Hölder and Young inequalities to estimate this term in a convenient way. Indeed, using convexity inequalities, it is simple to show that

$$
\left\||X|^{3} \nabla R\right\|_{L^{2}}^{2}+\left\||X|^{2}(X . \nabla R)\right\|_{L^{2}}^{2} \leq C\left\||X|^{4} \nabla R\right\|_{L^{2}}^{2}+C\|\nabla R\|_{L^{2}}^{2}
$$

and

$$
\alpha e^{-\tau}\left\||X|^{3} \Delta R\right\|_{L^{2}}^{2} \leq C \alpha e^{-\tau}\left\||X|^{4} \Delta R\right\|_{L^{2}}^{2}+C \alpha e^{-\tau}\|\Delta R\|_{L^{2}}^{2} .
$$

Thus, if we take $\varepsilon \leq \alpha M \gamma\left(\frac{3}{2}-\theta\right)^{2}$, we get

$$
\begin{aligned}
& I_{4} \leq C M \gamma\left(\frac{3}{2}-\theta\right)^{2}\left(\alpha e^{-\tau}\left\||X|^{4} \nabla R\right\|_{L^{2}}^{2}+\alpha^{2} e^{-2 \tau}\left\||X|^{4} \Delta R\right\|_{L^{2}}^{2}\right) \\
& +C M \gamma\left(\frac{3}{2}-\theta\right)^{2}\left(\alpha e^{-\tau}\|\nabla R\|_{L^{2}}^{2}+\alpha^{2} e^{-2 \tau}\|\Delta R\|_{L^{2}}^{2}\right) .
\end{aligned}
$$

As for the $H^{2}$-estimate, we have to study separately $I_{1}$ and $I_{2}$. We begin with $I_{1}$, that we rewrite

$$
I_{1}=I_{1}^{1}+I_{1}^{2}+I_{1}^{3}
$$

where

$$
\begin{aligned}
& I_{1}^{1}=\left(U . \nabla\left(R-\alpha e^{-\tau} \Delta R\right),|X|^{8}\left(R-\alpha e^{-\tau} \Delta R\right)\right)_{L^{2}}, \\
& I_{1}^{2}=e^{-2 \tau}\left(V_{\infty} \cdot \nabla\left(\Omega_{\infty}-\alpha e^{-\tau} \Delta \Omega_{\infty}\right),|X|^{8}\left(R-\alpha e^{-\tau} \Delta R\right)\right)_{L^{2}}, \\
& I_{1}^{3}=e^{-\tau}\left(K . \nabla\left(\Omega_{\infty}-\alpha e^{-\tau} \Delta \Omega_{\infty}\right),|X|^{8}\left(R-\alpha e^{-\tau} \Delta R\right)\right)_{L^{2}} .
\end{aligned}
$$

Using an integration by parts, the fact that $\operatorname{div} U=0$ and the Hölder inequalities, one has

$$
\begin{aligned}
I_{1}^{1} & =\frac{1}{2} \int_{\mathbb{R}^{3}}|X|^{8} U(X) \cdot \nabla\left(\left|R(X)-\alpha e^{-\tau} \Delta R(X)\right|^{2}\right) d X \\
& =-4 \int_{\mathbb{R}^{3}}|X|^{6}(X \cdot U(X))\left|R(X)-\alpha e^{-\tau} \Delta R(X)\right|^{2} d X \\
& \leq C\|U\|_{L^{\infty}}\left(\left\||X|^{7 / 2} R\right\|_{L^{2}}^{2}+\alpha^{2} e^{-2 \tau}\left\||X|^{7 / 2} \Delta R\right\|_{L^{2}}^{2}\right) .
\end{aligned}
$$


The inequalities (2.18) with $p=2, q=6$ and $\eta=\frac{1}{2}$ and (4.5) and the continuous injection of $H^{1}\left(\mathbb{R}^{3}\right)$ into $L^{6}\left(\mathbb{R}^{3}\right)$ imply

$$
\begin{aligned}
I_{1}^{1} & \leq C\|W\|_{L^{2}}^{1 / 2}\|W\|_{L^{6}}^{1 / 2}\left(\|R\|_{L^{2}}^{2}+\left\||X|^{4} R\right\|_{L^{2}}^{2}+\alpha^{2} e^{-2 \tau}\|\Delta R\|_{L^{2}}^{2}\right. \\
& \left.+\alpha^{2} e^{-2 \tau}\left\||X|^{4} \Delta R\right\|_{L^{2}}^{2}\right) \\
& \leq C\|W\|_{H^{1}}\left(\|R\|_{L^{2}}^{2}+\left\||X|^{4} R\right\|_{L^{2}}^{2}+\alpha^{2} e^{-2 \tau}\|\Delta R\|_{L^{2}}^{2}+\alpha^{2} e^{-2 \tau}\left\||X|^{4} \Delta R\right\|_{L^{2}}^{2}\right) \\
& \leq C M^{1 / 2} \gamma^{1 / 2}\left(\frac{3}{2}-\theta\right)\left(\|R\|_{L^{2}}^{2}+\left\||X|^{4} R\right\|_{L^{2}}^{2}\right. \\
& \left.+\alpha^{2} e^{-2 \tau}\|\Delta R\|_{L^{2}}^{2}+\alpha^{2} e^{-2 \tau}\left\||X|^{4} \Delta R\right\|_{L^{2}}^{2}\right) .
\end{aligned}
$$

Because of the smoothness of $\Omega_{\infty}, I_{1}^{2}$ is a little easier to estimate. Indeed using once more the inequalities (2.18) and (4.6) and the Hölder and Young inequalities, we get

$$
\begin{aligned}
I_{1}^{2} & \leq C e^{-2 \tau}\left\|V_{\infty}\right\|_{L^{\infty}}\left(\left\||X|^{4} \nabla \Omega_{\infty}\right\|_{L^{2}}+\alpha e^{-\tau}\left\||X|^{4} \nabla \Delta \Omega_{\infty}\right\|_{L^{2}}\right) \\
& \leq C|b| e^{-2 \tau}\left\|\Omega_{\infty}\right\|_{L^{2}}^{1 / 2}\left\|\Omega_{\infty}\right\|_{L^{6}}^{1 / 2}\left(\left\||X|^{4} R\right\|_{L^{2}}+\alpha\left\|_{L^{2}}+\alpha e^{-\tau}\right\||X|^{4} \Delta R \|_{L^{2}}\right) \\
& \leq C|b|^{2} e^{-2 \tau}\left(\left\||X|^{4} R\right\|_{L^{2}}+\alpha e^{-\tau}\left\||X|_{L^{2}}^{4} \Delta R\right\|_{L^{2}}\right) \\
& \leq \mu\left\||X|^{4} R\right\|_{L^{2}}^{2}+\mu \alpha^{2} e^{-2 \tau}\left\||X|^{4} \Delta R\right\|_{L^{2}}^{2}+\frac{C M^{2} \gamma^{2}\left(\frac{3}{2}-\theta\right)^{4}}{\mu} e^{-4 \tau} .
\end{aligned}
$$

Likewise, we get

$$
\begin{aligned}
I_{1}^{3} & \leq C|b| e^{-\tau}\|K\|_{L^{\infty}}\left(\left\||X|^{4} R\right\|_{L^{2}}+\alpha e^{-\tau}\left\||X|^{4} \Delta R\right\|_{L^{2}}\right) \\
& \leq C|b| e^{-\tau}\|R\|_{H^{1}}\left(\left\||X|^{4} R\right\|_{L^{2}}+\alpha e^{-\tau}\left\||X|^{4} \Delta R\right\|_{L^{2}}\right) \\
& \leq C M^{1 / 2} \gamma^{1 / 2}\left(\frac{3}{2}-\theta\right)\left(\|R\|_{L^{2}}^{2}+\|\nabla R\|_{L^{2}}^{2}+\left\||X|^{4} R\right\|_{L^{2}}^{2}\right. \\
& \left.+\alpha^{2} e^{-2 \tau}\left\||X|^{4} \Delta R\right\|_{L^{2}}^{2}\right) .
\end{aligned}
$$

Finally, taking $T$ so that $\alpha e^{-\tau_{0}}=\frac{\alpha}{T} \leq 1$, we have

$$
\begin{aligned}
& I_{1} \leq \mu\left\||X|^{4} R\right\|_{L^{2}}^{2}+\mu \alpha e^{-\tau}\left\||X|^{4} \Delta R\right\|_{L^{2}}^{2}+\frac{C M^{2} \gamma^{2}\left(\frac{3}{2}-\theta\right)^{4}}{\mu} e^{-4 \tau} \\
& +C M^{1 / 2} \gamma^{1 / 2}\left(\frac{3}{2}-\theta\right)\left(\|R\|_{L^{2}}^{2}+\|\nabla R\|_{L^{2}}^{2}+\|\Delta R\|_{L^{2}}^{2}\right. \\
& \left.+\left\||X|^{4} R\right\|_{L^{2}}^{2}+\alpha e^{-\tau}\left\||X|^{4} \Delta R\right\|_{L^{2}}^{2}\right)
\end{aligned}
$$


It remains to bound $I_{2}$, which is the hardest term to estimate. Like for $I_{1}$, we rewrite it

$$
I_{2}=I_{2}^{1}+I_{2}^{2}+I_{2}^{3}+I_{2}^{4}
$$

where

$$
\begin{aligned}
& I_{2}^{1}=e^{-\tau}\left(\left(R-\alpha e^{-\tau} \Delta R\right) \cdot \nabla V_{\infty},|X|^{8}\left(R-\alpha e^{-\tau} \Delta R\right)\right)_{L^{2}}, \\
& I_{2}^{2}=\left(\left(R-\alpha e^{-\tau} \Delta R\right) \cdot \nabla K,|X|^{8}\left(R-\alpha e^{-\tau} \Delta R\right)\right)_{L^{2}}, \\
& I_{2}^{3}=e^{-2 \tau}\left(\left(\Omega_{\infty}-\alpha e^{-\tau} \Delta \Omega_{\infty}\right) \cdot \nabla V_{\infty},|X|^{8}\left(R-\alpha e^{-\tau} \Delta R\right)\right)_{L^{2}} \\
& I_{2}^{4}=e^{-\tau}\left(\left(\Omega_{\infty}-\alpha e^{-\tau} \Delta \Omega_{\infty}\right) \cdot \nabla K,|X|^{8}\left(R-\alpha e^{-\tau} \Delta R\right)\right)_{L^{2}} .
\end{aligned}
$$

Using the inequality (2.18) and the smoothness of $\Omega_{\infty}$, we get

$$
\begin{aligned}
I_{2}^{1} & \leq C e^{-\tau}\left\|\nabla V_{\infty}\right\|_{L^{\infty}}\left(\left\||X|^{4} R\right\|_{L^{2}}^{2}+\alpha^{2} e^{-2 \tau}\left\||X|^{4} \Delta R\right\|_{L^{2}}^{2}\right) \\
& \leq C e^{-\tau}\left\|\nabla \Omega_{\infty}\right\|_{L^{2}}^{1 / 2}\left\|\nabla \Omega_{\infty}\right\|_{L^{6}}^{1 / 2}\left(\left\||X|^{4} R\right\|_{L^{2}}^{2}+\alpha^{2} e^{-2 \tau}\left\||X|^{4} \Delta R\right\|_{L^{2}}^{2}\right) \\
& \leq C|b|\left(\left\||X|^{4} R\right\|_{L^{2}}^{2}+\alpha^{2} e^{-2 \tau}\left\||X|^{4} \Delta R\right\|_{L^{2}}^{2}\right) \\
& \leq C M^{1 / 2} \gamma^{1 / 2}\left(\frac{3}{2}-\theta\right)\left(\left\||X|^{4} R\right\|_{L^{2}}^{2}+\alpha^{2} e^{-2 \tau}\left\||X|^{4} \Delta R\right\|_{L^{2}}^{2}\right) .
\end{aligned}
$$

We now estimate $I_{2}^{2}$. We recall the notation $\delta=M \gamma\left(\frac{3}{2}-\theta\right)^{2}$. Using again the inequality (2.18), the inequality (4.6) and the continuous injection of $H^{1}\left(\mathbb{R}^{3}\right)$ into $L^{6}\left(\mathbb{R}^{3}\right)$, one has

$$
\begin{aligned}
I_{2}^{2} & \leq\|\nabla K\|_{L^{\infty}}\left(\left\||X|^{4} R\right\|_{L^{2}}^{2}+\alpha^{2} e^{-2 \tau}\left\||X|^{4} \Delta R\right\|_{L^{2}}^{2}\right) \\
& \leq C\|\nabla R\|_{L_{2}}^{1 / 2}\|\nabla R\|_{L_{6}}^{1 / 2}\left(\left\||X|^{4} R\right\|_{L^{2}}^{2}+\alpha^{2} e^{-2 \tau}\left\||X|^{4} \Delta R\right\|_{L^{2}}^{2}\right) \\
& \leq C \delta^{1 / 4}\|\nabla R\|_{H^{1}}^{1 / 2}\left(\left\||X|^{4} R\right\|_{L^{2}}^{2}+\alpha^{2} e^{-2 \tau}\left\||X|^{4} \Delta R\right\|_{L^{2}}^{2}\right) \\
& \leq C \delta^{1 / 2}\left(\left\||X|^{4} R\right\|_{L^{2}}^{2}+\alpha^{2} e^{-2 \tau}\left\||X|^{4} \Delta R\right\|_{L^{2}}^{2}\right) \\
& +C \delta^{1 / 4}\|\Delta R\|_{L^{2}}^{1 / 2}\left(\left\||X|^{4} R\right\|_{L^{2}}^{2}+\alpha^{2} e^{-2 \tau}\left\||X|^{4} \Delta R\right\|_{L^{2}}^{2}\right) \\
& \leq C \delta^{1 / 2}\left(\left\||X|^{4} R\right\|_{L^{2}}^{2}+\alpha^{2} e^{-2 \tau}\left\||X|^{4} \Delta R\right\|_{L^{2}}^{2}\right)+C \delta^{1 / 4}\|\Delta R\|_{L^{2}}^{1 / 2}\left\||X|^{4} R\right\|_{L^{2}}^{2} \\
& +C \delta^{1 / 2} \alpha^{7 / 4} e^{-\frac{7 \tau}{4}}\left\||X|^{4} \Delta R\right\|_{L^{2}}^{2} \cdot
\end{aligned}
$$


To finish the estimate of $I_{2}^{2}$, we use the convexity inequality $a b \leq \frac{3}{4} a^{\frac{4}{3}}+\frac{1}{4} b^{4}$ and the condition (4.6). We obtain

$$
\begin{aligned}
I_{2}^{2} & \leq C \delta^{1 / 2}\left(\left\||X|^{4} R\right\|_{L^{2}}^{2}+\alpha^{2} e^{-2 \tau}\left\||X|^{4} \Delta R\right\|_{L^{2}}^{2}\right) \\
& +C \delta^{1 / 4}\left(\|\Delta R\|_{L^{2}}^{2}+\left\||X|^{4} R\right\|_{L^{2}}^{8 / 3}\right) \\
& +C \delta^{1 / 2} \alpha^{7 / 4} e^{-\frac{7 \tau}{4}}\left\||X|^{4} \Delta R\right\|_{L^{2}}^{2} \\
& \leq C \delta^{1 / 2}\left(\left\||X|^{4} R\right\|_{L^{2}}^{2}+\alpha^{2} e^{-2 \tau}\left\||X|^{4} \Delta R\right\|_{L^{2}}^{2}\right) \\
& +C \delta^{1 / 4}\|\Delta R\|_{L^{2}}^{2}+C \delta^{7 / 12}\left\||X|^{4} R\right\|_{L^{2}}^{2} \\
& +C \delta^{1 / 2} \alpha^{7 / 4} e^{-\frac{7 \tau}{4}}\left\||X|^{4} \Delta R\right\|_{L^{2}}^{2} \cdot
\end{aligned}
$$

Consequently, if we assume $\gamma \leq 1$ and $\left(\frac{3}{2}-\theta\right) \leq 1$, one has

$$
I_{2}^{2} \leq C M^{7 / 12} \gamma^{1 / 4}\left(\frac{3}{2}-\theta\right)^{1 / 2}\left(\left\||X|^{4} R\right\|_{L^{2}}^{2}+\|\Delta R\|_{L^{2}}^{2}+\alpha^{2} e^{-2 \tau}\left\||X|^{4} \Delta R\right\|_{L^{2}}^{2}\right)
$$

It it easier bound $I_{2}^{3}$. Indeed, the inequality (2.19) and the inequality (4.6) imply

$$
\begin{aligned}
I_{2}^{3} & \leq C e^{-2 \tau}\left\|\Omega_{\infty}-\alpha e^{-\tau} \Delta \Omega_{\infty}\right\|_{L^{\infty}}\left\|\nabla V_{\infty}\right\|_{L^{2}}\left(\left\||X|^{4} R\right\|_{L^{2}}+\alpha e^{-\tau}\left\||X|^{4} \Delta R\right\|_{L^{2}}\right) \\
& \leq C|b| e^{-2 \tau}\left\|\Omega_{\infty}\right\|_{L^{2}}\left(\left\||X|^{4} R\right\|_{L^{2}}+\alpha e^{-\tau}\left\||X|^{4} \Delta R\right\|_{L^{2}}\right) \\
& \leq C|b|^{2} e^{-2 \tau}\left(\left\||X|^{4} R\right\|_{L^{2}}+\alpha e^{-\tau}\left\||X|^{4} \Delta R\right\|_{L^{2}}\right) \\
& \leq \mu\left\||X|^{4} R\right\|_{L^{2}}^{2}+\mu \alpha^{2} e^{-2 \tau}\left\||X|^{4} \Delta R\right\|_{L^{2}}^{2}+\frac{C M^{2} \gamma^{2}\left(\frac{3}{2}-1\right)^{4}}{\mu} e^{-4 \tau} .
\end{aligned}
$$

Likewise, we obtain

$$
\begin{aligned}
I_{2}^{4} & \leq C e^{-\tau}\left\|\Omega_{\infty}-\alpha e^{-\tau} \Delta \Omega_{\infty}\right\|_{L^{\infty}}\|\nabla K\|_{L^{2}}\left(\left\||X|^{4} R\right\|_{L^{2}}+\alpha e^{-\tau}\left\||X|^{4} \Delta R\right\|_{L^{2}}\right) \\
& \leq C|b| e^{-\tau}\|R\|_{L^{2}}\left(\left\||X|^{4} R\right\|_{L^{2}}+\alpha e^{-\tau}\left\||X|^{4} \Delta R\right\|_{L^{2}}\right) \\
& \leq C M^{1 / 2} \gamma^{1 / 2}\left(\frac{3}{2}-1\right)\left(\|R\|_{L^{2}}^{2}+\left\||X|^{4} R\right\|_{L^{2}}^{2}+\alpha^{2} e^{-2 \tau}\left\||X|^{4} \Delta R\right\|_{L^{2}}^{2}\right) .
\end{aligned}
$$

Thus, taking $T_{0}$ large enough so that $\alpha e^{-\tau_{0}}=\frac{\alpha}{T} \leq 1$, the following inequality holds:

$$
\begin{aligned}
& I_{2} \leq \mu\left(\left\||X|^{4} R\right\|_{L^{2}}^{2}+\alpha e^{-\tau}\left\||X|^{4} \Delta R\right\|_{L^{2}}^{2}\right)+\frac{C M^{2} \gamma^{2}\left(\frac{3}{2}-1\right)^{4}}{\mu} e^{-4 \tau} \\
& +C M \gamma^{1 / 4}\left(\frac{3}{2}-1\right)^{1 / 2}\left(\|R\|_{L^{2}}^{2}+\|\Delta R\|_{L^{2}}^{2}+\left\||X|^{4} R\right\|_{L^{2}}^{2}+\alpha e^{-\tau}\left\||X|^{4} \Delta R\right\|_{L^{2}}^{2}\right)
\end{aligned}
$$


Combining the equality (4.42) together with the inequalities (4.43), (4.44), (4.45) and (4.46) and taking $T_{0}$ big enough compared to $\alpha$, we have (4.47)

$$
\begin{aligned}
& \frac{1}{2} \partial_{\tau}\left(\left\||X|^{4}\left(R-\alpha e^{-\tau} \Delta R\right)\right\|_{L^{2}}^{2}\right)+\frac{7}{4}\left\||X|^{4} R\right\|_{L^{2}}^{2}+\left(1+\frac{7 \alpha}{2} e^{-\tau}\right)\left\||X|^{4} \nabla R\right\|_{L^{2}}^{2} \\
& +\left(\alpha e^{-\tau}+\frac{7 \alpha^{2}}{4} e^{-2 \tau}\right)\left\||X|^{4} \Delta R\right\|_{L^{2}}^{2}-108 \alpha e^{-\tau}\left\||X|^{3} R\right\|_{L^{2}}^{2} \leq \\
& C\left(M \gamma^{1 / 4}\left(\frac{3}{2}-\theta\right)^{1 / 2}+\mu\right) \\
& \left(\left\||X|^{4} R\right\|_{L^{2}}^{2}+\alpha e^{-\tau}\left\||X|^{4} \nabla R\right\|_{L^{2}}^{2}+\alpha e^{-\tau}\left\||X|^{4} \Delta R\right\|_{L^{2}}^{2}\right) \\
& +36\left\||X|^{3} R\right\|_{L^{2}}^{2}+C M \gamma^{1 / 4}\left(\frac{3}{2}-\theta\right)^{1 / 2}\left(\|R\|_{L^{2}}^{2}+\|\nabla R\|_{L^{2}}^{2}+\|\Delta R\|_{L^{2}}^{2}\right) \\
& +\frac{C M^{2} \gamma\left(\frac{3}{2}-\theta\right)^{2}}{\mu} e^{-4 \tau} .
\end{aligned}
$$

Integrating several times by parts, it is easy to check that

$$
E_{4}=\frac{1}{2}\left\||X|^{4} R\right\|_{L^{2}}^{2}+\frac{\alpha^{2}}{2} e^{-2 \tau}\left\||X|^{4} \Delta R\right\|_{L^{2}}^{2}+\alpha e^{-\tau}\left\||X|^{4} \nabla R\right\|_{L^{2}}^{2}-36 \alpha e^{-\tau}\left\||X|^{3} R\right\|_{L^{2}}^{2} .
$$

Consequently, the inequality (4.47) becomes

$$
\begin{aligned}
& \partial_{\tau} E_{5}+3 E_{5}+\frac{1}{4}\left\||X|^{4} R\right\|_{L^{2}}^{2}+\left(1+\frac{\alpha}{2} e^{-\tau}\right)\left\||X|^{4} \nabla R\right\|_{L^{2}}^{2} \\
& +\left(\alpha e^{-\tau}+\frac{\alpha^{2}}{4} e^{-2 \tau}\right)\left\||X|^{4} \Delta R\right\|_{L^{2}}^{2} \leq \\
& C\left(M \gamma^{1 / 4}\left(\frac{3}{2}-\theta\right)^{1 / 2}+\mu\right) \\
& \left(\left\||X|^{4} R\right\|_{L^{2}}^{2}+\alpha e^{-\tau}\left\||X|^{4} \nabla R\right\|_{L^{2}}^{2}+\alpha e^{-\tau}\left\||X|^{4} \Delta R\right\|_{L^{2}}^{2}\right) \\
& +36\left\||X|^{3} R\right\|_{L^{2}}^{2}+C M \gamma^{1 / 4}\left(\frac{3}{2}-\theta\right)^{1 / 2}\left(\|R\|_{L^{2}}^{2}+\|\nabla R\|_{L^{2}}^{2}+\|\Delta R\|_{L^{2}}^{2}\right) \\
& +\frac{C M^{2} \gamma\left(\frac{3}{2}-\theta\right)^{2}}{\mu} e^{-4 \tau} .
\end{aligned}
$$

Thus, taking $\gamma_{0}$ and $\mu$ small enough, we obtain

$$
\begin{aligned}
& \partial_{\tau} E_{5}+3 E_{5}+\frac{1}{8}\left\||X|^{4} R\right\|_{L^{2}}^{2}+\left(\frac{\alpha}{2} e^{-\tau}+\frac{\alpha^{2}}{4} e^{-2 \tau}\right)\left\||X|^{4} \Delta R\right\|_{L^{2}}^{2} \leq 36\left\||X|^{3} R\right\|_{L^{2}}^{2} \\
& +C M \gamma^{1 / 4}\left(\frac{3}{2}-\theta\right)^{1 / 2}\left(\|R\|_{L^{2}}^{2}+\|\nabla R\|_{L^{2}}^{2}+\|\Delta R\|_{L^{2}}^{2}\right)+C M^{2} \gamma\left(\frac{3}{2}-\theta\right)^{2} e^{-4 \tau} .
\end{aligned}
$$


Using Hölder and the convexity inequality $a b \leq \frac{1}{4} a^{4}+\frac{3}{4} b^{\frac{4}{3}}$, a simple computation leads to

$$
\left\||X|^{3} R\right\|_{L^{2}}^{2} \leq \frac{3 \mu^{4 / 3}}{4}\left\||X|^{4} R\right\|_{L^{2}}^{2}+\frac{1}{4 \mu^{4}}\|R\|_{L^{2}}^{2}
$$

for all $\mu>0$.

Using this inequality with $\mu$ small enough, we finally obtain

$$
\begin{aligned}
& \partial_{\tau} E_{5}+3 E_{5}+\frac{1}{16}\left\||X|^{4} R\right\|_{L^{2}}^{2}+\left(\frac{\alpha}{2} e^{-\tau}+\frac{\alpha^{2}}{4} e^{-2 \tau}\right)\left\||X|^{4} \Delta R\right\|_{L^{2}}^{2} \leq K_{1}\|R\|_{L^{2}}^{2} \\
& +C M \gamma^{1 / 4}\left(\frac{3}{2}-\theta\right)^{1 / 2}\left(\|R\|_{L^{2}}^{2}+\|\nabla R\|_{L^{2}}^{2}+\|\Delta R\|_{L^{2}}^{2}\right)+C M^{2} \gamma\left(\frac{3}{2}-\theta\right)^{2} e^{-4 \tau}
\end{aligned}
$$

where $K_{1}$ is a positive constant.

This lemma, combined with the inequality (4.35) enables to finish the $H^{2}(4)$ estimate of $R$. We define the functional

$$
E_{6}=K E_{4}+E_{5},
$$

with $K$ some large positive constant that will be made more precise later.

Inequalities (4.35) and (4.41) show that one has

$$
\begin{aligned}
& \partial_{\tau} E_{6}+2 \theta E_{6}+10 K\left\|(-\Delta)^{-\left(\theta-\frac{1}{4}\right)} R\right\|_{L^{2}}^{2}+\frac{K}{2}\|\nabla R\|_{L^{2}}^{2}+\frac{K}{4}\|\Delta R\|_{L^{2}}^{2} \\
& +\frac{1}{16}\left\||X|^{4} R\right\|_{L^{2}}^{2}+\frac{\alpha^{2}}{4} e^{-2 \tau}\left\||X|^{4} \Delta R\right\|_{L^{2}}^{2} \leq \\
& K_{1}\|R\|_{L^{2}}^{2}+C M^{2} \gamma^{1 / 4}\left(\|R\|_{L^{2}}^{2}+\|\nabla R\|_{L^{2}}^{2}+\|\Delta R\|_{L^{2}}^{2}+\left\||X|^{4} R\right\|_{L^{2}}^{2}\right) \\
& +C M^{2} \gamma\left(\frac{3}{2}-\theta\right)^{2} e^{-\frac{7 \tau}{2}} .
\end{aligned}
$$

Interpolating again $\|R\|_{L^{2}}^{2}$ between $\left\|(-\Delta)^{-\left(\theta-\frac{1}{4}\right)} R\right\|_{L^{2}}^{2}$ and $\|\nabla R\|_{L^{2}}^{2}$ and taking $K$ and $\gamma_{0}$ respectively sufficiently large and small, we get

$$
\partial_{\tau} E_{6}+2 \theta E_{6} \leq C M^{2} \gamma\left(\frac{3}{2}-\theta\right)^{2} e^{-\frac{7 \tau}{2}} .
$$

\section{Proof of Theorem 2.2}

Theorem 2.2 for approximate solutions. In this section, under the condition (2.10), we show that the solutions of the approximate system (4.1) are actually global in time and that the inequality (2.11) of Theorem 2.2 holds for these solutions. To get this result, we make use of the energy estimates that we have obtained in Section 4. The following theorem is a copy of Theorem 2.2 for the solutions of the regularized system (4.1).

THEOREM 5.1. Let $\theta$ be a fixed positive constant such that $1<\theta<\frac{3}{2}$, $\varepsilon$ be a positive constant and $W_{0} \in \mathbb{H}^{2}(4)$. There exist three positive constants $\gamma_{0}=\gamma_{0}(\alpha)$, 
$\varepsilon=\varepsilon_{0}(\alpha)$ and $T=T_{0}(\alpha)$ such that if $T \geq T_{0}, \varepsilon \leq \varepsilon_{0}$ and there exists a positive constant $\gamma \leq \gamma_{0}$ such that $W_{0} \in \mathbb{H}^{2}(4)$ satisfies the condition

$\left\|W_{0}\right\|_{L^{2}(4)}^{2}+\left\|\nabla W_{0}\right\|_{L^{2}}^{2}+\alpha e^{-\tau_{0}}\left\|\Delta W_{0}\right\|_{L^{2}}^{2}+\alpha^{2} e^{-2 \tau_{0}}\left\||X|^{4} \Delta W_{0}\right\|_{L^{2}}^{2} \leq \gamma\left(\frac{3}{2}-\theta\right)^{2}$,

where $\tau_{0}=\log (T)$,

then there exist a unique solution $W_{\varepsilon} \in C^{1}\left(\left(\tau_{0},+\infty\right), \mathbb{H}^{1}(4)\right) \cap C^{0}\left(\left(\tau_{0},+\infty\right), \mathbb{H}^{3}(4)\right)$ to the system (4.1) and a positive constant $C=C\left(\alpha, \tau_{0}\right)$ such that, for all $\tau \geq \tau_{0}$,

$$
\left\|\left(I d-\alpha e^{-\tau} \Delta\right)\left(W_{\varepsilon}(\tau)-e^{-\tau} \sum_{i=1}^{3} b_{i} f_{i}\right)\right\|_{L^{2}(4)} \leq C \gamma^{1 / 2}\left(\frac{3}{2}-\theta\right) e^{-\theta \tau},
$$

where $b_{i}=\int_{\mathbb{R}^{3}} p_{i}(X) \cdot W_{0}(X) d X$.

In order to prove this theorem, we use the energy estimates that we established in Section 4. To obtain the inequality (5.2), we need the energy functional $E_{6}$ to be equivalent to the $H^{2}(4)$-norm of $R_{\varepsilon}$. If we take $K$ large enough in the definition (4.52) of $E_{6}$, then the next lemma holds.

LEMmA 5.2. Let $R_{\varepsilon} \in C^{1}\left(\left(\tau_{0},+\infty\right), \mathbb{H}^{1}(4)\right) \cap C^{0}\left(\left(\tau_{0},+\infty\right), \mathbb{H}^{3}(4)\right)$ and $E_{6}$ be the energy functional defined by (4.52). There exists $K_{0}$ such that, if $K \geq K_{0}$, then there exists a positive constant $C$ such that

$$
E_{6}(\tau) \leq C\left(\left\|R_{\varepsilon}\right\|_{L^{2}(4)}^{2}+\left\|\nabla R_{\varepsilon}\right\|_{L^{2}}^{2}+\alpha e^{-\tau}\left\|\Delta R_{\varepsilon}\right\|_{L^{2}}^{2}+\alpha^{2} e^{-2 \tau}\left\||X|^{4} \Delta R_{\varepsilon}\right\|_{L^{2}(4)}^{2}\right),
$$

$$
C\left(\left\|R_{\varepsilon}\right\|_{L^{2}(4)}^{2}+\left\|\nabla R_{\varepsilon}\right\|_{L^{2}}^{2}+\alpha e^{-\tau}\left\|\Delta R_{\varepsilon}\right\|_{L^{2}}^{2}+\alpha^{2} e^{-2 \tau}\left\||X|^{4} \Delta R_{\varepsilon}\right\|_{L^{2}(4)}^{2}\right) \leq E_{6}(\tau) .
$$

Proof. The inequalities (5.3) and (5.4) come directly from the definition of $E_{6}$ and the interpolation inequality (4.16).

Proof of Theorem 5.1. Let $\theta$ be a fixed constant such that $1<\theta<\frac{3}{2}$, $W_{0} \in \mathbb{H}^{2}(4)$ and $W_{\varepsilon} \in C^{1}\left(\left(\tau_{0},+\infty\right), \mathbb{H}^{1}(4)\right) \cap C^{0}\left(\left(\tau_{0},+\infty\right), \mathbb{H}^{3}(4)\right)$ be the solution of the system (4.1) given by Theorem 3.1, with initial data $W_{0}$. Let $T$ and $K$ be sufficiently large so that they satisfy the conditions of the lemmas 4.5, 4.6, 4.7 and 4.9 and assume that the initial data $W_{0}$ satisfy the condition (2.10) for some $\gamma>0$ which will be made more precise later. We decompose $W_{\varepsilon}$ such that

$$
W_{\varepsilon}=e^{-\tau} \Omega_{\infty}+R_{\varepsilon}
$$

where $\Omega_{\infty}=\sum_{i=1}^{3} b_{i} f_{i}, b_{i}=\int_{\mathbb{R}^{3}} p_{i}(X) . W_{0}(X) d X$ and $\left\{f_{1}, f_{2}, f_{3}\right\}$ is the basis of the eigenspace of $\mathcal{L}$ associated to the eigenvalue -1 , given by (2.1).

Let $M$ be a positive constant such that $M>2$ that will be made more precise later and $\tau_{\varepsilon}^{*} \in\left[\tau_{0}, \tau_{\varepsilon}\right]$ be the biggest positive time such that the inequality (4.5) holds. 
We take $\gamma$ and $\varepsilon$ sufficiently small so that the lemmas 4.5, 4.6, 4.7 and 4.9 hold. According to the inequality (4.53), one has, for all $\tau \in\left[\tau_{0}, \tau_{\varepsilon}^{*}\right)$,

$$
\partial_{\tau}\left(E_{6}(\tau) e^{2 \theta \tau}\right) \leq C M^{2} \gamma\left(\frac{3}{2}-\theta\right)^{2} e^{-\left(\frac{7}{2}-2 \theta\right) \tau}
$$

Integrating in time the previous inequality between $\tau_{0}$ and $\tau \in\left[\tau_{0}, \tau_{\varepsilon}^{*}\right)$, we get

$$
E_{6}(\tau) \leq E_{6}\left(\tau_{0}\right) e^{-2 \theta\left(\tau-\tau_{0}\right)}+C M^{2} \gamma\left(\frac{3}{2}-\theta\right)^{2} e^{-\frac{7 \tau_{0}}{2}}\left(e^{-2 \theta\left(\tau-\tau_{0}\right)}-e^{-\frac{7}{2}\left(\tau-\tau_{0}\right)}\right)
$$

Arguing like in the proof of Lemma 4.1 and using the inequality (5.3), we can show that

$$
E_{6}\left(\tau_{0}\right) \leq C \gamma\left(\frac{3}{2}-\theta\right)^{2}
$$

which implies

$$
E_{6}(\tau) \leq C \gamma\left(\frac{3}{2}-\theta\right)^{2}+C M^{2} \gamma\left(\frac{3}{2}-\theta\right)^{2} e^{-\frac{7 \tau_{0}}{2}}
$$

According to the inequalities (5.4) and (4.6), one has, for all $\tau \in\left[\tau_{0}, \tau_{\varepsilon}^{*}\right)$,

$$
\begin{aligned}
& |b|^{2}+\left\|R_{\varepsilon}\right\|_{L^{2}(4)}^{2}+\left\|\nabla R_{\varepsilon}\right\|_{L^{2}}^{2}+\alpha e^{-\tau}\left\|\Delta R_{\varepsilon}\right\|_{L^{2}}^{2}+\alpha^{2} e^{-2 \tau}\left\||X|^{4} \Delta R_{\varepsilon}\right\|^{2} \leq \\
& C \gamma\left(\frac{3}{2}-\theta\right)^{2}+C M^{2} \gamma\left(\frac{3}{2}-\theta\right)^{2} e^{-\frac{7 \tau_{0}}{2}} .
\end{aligned}
$$

Recalling that $W_{\varepsilon}=\sum_{i=1}^{3} b_{i} f_{i}+R_{\varepsilon}$, we get

$$
\begin{aligned}
& \left\|W_{\varepsilon}\right\|_{L^{2}(4)}^{2}+\left\|\nabla W_{\varepsilon}\right\|_{L^{2}}^{2}+\alpha e^{-\tau}\left\|\Delta W_{\varepsilon}\right\|_{L^{2}}^{2}+\alpha^{2} e^{-2 \tau}\left\||X|^{4} \Delta W_{\varepsilon}\right\|^{2} \leq \\
& C_{1} \gamma\left(\frac{3}{2}-\theta\right)^{2}+C_{2} M^{2} \gamma\left(\frac{3}{2}-\theta\right)^{2} e^{-\frac{7 \tau_{0}}{2}},
\end{aligned}
$$

where $C_{1}$ and $C_{2}$ are two positive constants.

We take $M$ sufficiently large so that $C_{1} \leq \frac{M}{4}$ and $\tau_{0}=\ln (T)$ sufficiently large so that $C_{2} M^{2} e^{-\frac{7 \tau_{0}}{2}} \leq \frac{M}{4}$, we obtain, for all $\tau \in\left[\tau_{0}, \tau_{\varepsilon}^{*}\right)$,

$$
\left\|W_{\varepsilon}\right\|_{L^{2}(4)}^{2}+\left\|\nabla W_{\varepsilon}\right\|_{L^{2}}^{2}+\alpha e^{-\tau}\left\|\Delta W_{\varepsilon}\right\|_{L^{2}}^{2}+\alpha^{2} e^{-2 \tau}\left\||X|^{4} \Delta W_{\varepsilon}\right\|^{2} \leq \frac{M \gamma\left(\frac{3}{2}-\theta\right)^{2}}{2} .
$$

In particular, the inequality (5.8) shows that $\tau_{\varepsilon}^{*}=\tau_{\varepsilon}$. Furthermore, letting $\tau$ tend to $\tau_{\varepsilon}$, we see that if $\tau_{\varepsilon}$ is finite, then the $H^{1}(4)$ norm of $W_{\varepsilon}$ stay bounded on $\left[\tau, \tau_{\varepsilon}\right)$. According to the proof of Theorem 3.1, it implies in particular that one can extend the interval of definition of $W_{\varepsilon}$ over $\tau_{\varepsilon}$. Consequently, we have necessarily $\tau_{\varepsilon}=+\infty$. In addition, going back to the inequality (5.6) and applying the inequality (5.4) of Lemma 5.2, we see that the inequality (5.2) holds. 
Existence of weak solutions in $\mathbb{H}^{2}(4)$. In this section, we show that there exists a weak solution to the system (1.6) belonging to the space $C^{0}\left(\left[\tau_{0},+\infty\right), \mathbb{H}^{2}(4)\right)$. To this end, we show that, when $\varepsilon$ tends to $0, W_{\varepsilon}$ tends to a divergence free vector $W$ which satsifies (1.6) in a weak sense. Let $\left(\varepsilon_{n}\right)_{n \in \mathbb{N}}$ be a sequence of positive terms which tends to 0 . Let $W_{\varepsilon_{n}} \in C^{1}\left(\left(\tau_{0},+\infty\right), \mathbb{H}^{1}(4)\right) \cap C^{0}\left(\left(\tau_{0},+\infty\right), \mathbb{H}^{3}(4)\right)$ be the global solution of (4.1) given by Theorem 5.1, with initial data $W_{0}$. Let $\mathcal{O}$ be a bounded open set of $\mathbb{R}^{3}$. For $s \in \mathbb{R}^{+}, H^{s}(\mathcal{O})$ denotes the restriction of the Sobolev space $H^{s}\left(\mathbb{R}^{3}\right)$ on $\mathcal{O}$. For $s \geq 1$, we define also the space

$$
H_{0}^{s}(\mathcal{O})=\left\{u \in H^{s}(\mathcal{O}): u_{\mid \partial \mathcal{O}=0}\right\}
$$

Let $\tau_{1}$ be a fixed positive time such that $\tau_{1}>\tau_{0}$. Due to the boundedness property of $W_{\varepsilon_{n}}$ in $L^{\infty}\left(\left[\tau_{0}, \tau_{1}\right], \mathbb{H}^{2}(4)\right)$ uniformly with respect to $n$, there exist $W \in L^{\infty}\left(\left[\tau_{0}, \tau_{1}\right], \mathbb{H}^{2}(4)\right)$ and a subsequence of $\varepsilon_{n}$ (that we still note $\varepsilon_{n}$ ) such that

$$
W_{\varepsilon_{n}} \rightarrow W \quad \text { weak }^{*} \text { in } \quad L^{\infty}\left(\left[\tau_{0}, \tau_{1}\right], H^{2}(\mathcal{O})^{3}\right)
$$

Since $W_{\varepsilon_{n}}$ is bounded in $L^{\infty}\left(\left[\tau_{0}, \tau_{1}\right], \mathbb{H}^{2}(4)\right)$, applying the operator $\left(I-\alpha e^{-\tau} \Delta\right)^{-1}$ to the first equality of $(4.1)$, it is quite easy to see that $\partial_{\tau} W_{\varepsilon_{n}}$ is bounded in $L^{\infty}\left(\left[\tau_{0}, T\right], L^{2}(\mathcal{O})^{3}\right)$ uniformly with respect to $n$. Consequently, $W_{\varepsilon_{n}}$ is equicontinuous in time on $L^{2}(\mathcal{O})^{3}$. Indeed, given $\sigma_{1}$ and $\sigma_{2}$ belonging to $\left[\tau_{0}, \tau_{1}\right]$, one has

$$
\begin{aligned}
\left\|W_{\varepsilon_{n}}\left(\sigma_{1}\right)-W_{\varepsilon_{n}}\left(\sigma_{2}\right)\right\|_{L^{2}(\mathcal{O})} & =\left\|\int_{\sigma_{2}}^{\sigma_{1}} \partial_{\tau} W_{\varepsilon_{n}}(s) d s\right\|_{L^{2}(\mathcal{O})} \\
& \leq\left|\int_{\sigma_{2}}^{\sigma_{1}}\left\|\partial_{\tau} W_{\varepsilon_{n}}(s)\right\|_{L^{2}(\mathcal{O})} d s\right| \\
& \leq\left|\sigma_{1}-\sigma_{2}\right| \max _{s \in\left[\tau_{0}, T\right]}\left\|\partial_{\tau} W_{\varepsilon_{n}}(s)\right\|_{L^{2}(\mathcal{O})} .
\end{aligned}
$$

Besides, for all $\tau \in\left[\tau_{0}, \tau_{1}\right]$, the set $\bigcup_{n \in \mathbb{N}} W_{\varepsilon_{n}}(\tau)$ is bounded in $H^{2}(\mathcal{O})^{3}$ and thus compact in $L^{2}(\mathcal{O})^{3}$. Applying the classical Arzela-Ascoli theorem, we conclude that

$$
W_{\varepsilon_{n}} \longrightarrow W \text { strongly in } \quad C^{0}\left(\left[\tau_{0}, \tau_{1}\right], L^{2}(\mathcal{O})^{3}\right)
$$

A classical interpolation inequality between $L^{2}$ and $H^{2}$ yields, for all $s<2$,

$$
W_{\varepsilon_{n}} \longrightarrow W \quad \text { strongly in } \quad C^{0}\left(\left[\tau_{0}, \tau_{1}\right], H^{s}(\mathcal{O})^{3}\right)
$$

The two identities (5.9) and (5.10) are sufficient to pass to the limit in the weak formulation of the system (4.1) and to show that $W$ is a weak solution of the system (1.6). More precisely, for every $\varphi \in C^{1}\left(\left[\tau_{0}, \tau_{1}\right], H_{0}^{1}(\mathcal{O})^{3}\right)$ such that $\operatorname{div} \varphi=0$, one 
has, for all $\tau \in\left[\tau_{0}, \tau_{1}\right]$,

$$
\begin{aligned}
& \int_{\mathcal{O}}\left(W(\tau)-\alpha e^{-\tau} \Delta W(\tau)\right) \cdot \varphi(\tau) d X+\int_{\tau_{0}}^{\tau} \int_{\mathcal{O}} \mathcal{L}(W(\sigma)) \cdot \varphi(\sigma) d X d \sigma \\
& +\int_{\tau_{0}}^{\tau} \int_{\mathcal{O}}\left(W(\sigma)-\alpha e^{-\sigma} \Delta W(\sigma)\right) \wedge U(\sigma) \cdot \operatorname{curl} \varphi(\sigma) d X d \sigma \\
& =\int_{\mathcal{O}}\left(W_{0}-\alpha e^{-\tau_{0}} \Delta W_{0}\right) \cdot \varphi\left(\tau_{0}\right) d X+\int_{\tau_{0}}^{\tau} \int_{\mathcal{O}}\left(W(\sigma)-\alpha e^{-\sigma} \Delta W(\sigma)\right) . \partial_{\tau} \varphi(\sigma) d X d \sigma \\
& +\int_{\tau_{0}}^{\tau} \int_{\mathcal{O}} \frac{3 \alpha}{2} e^{-\sigma} \Delta W(\sigma) \cdot \varphi(\sigma) d X d \sigma+\int_{\tau_{0}}^{\tau} \int_{\mathcal{O}} \frac{\alpha}{2} e^{-\sigma} \Delta W(\sigma)(X . \nabla \varphi(\sigma)) d X d \sigma .
\end{aligned}
$$

We only show that the non-linear term converges, using (5.9) and (5.10). The other ones are nearly obvious. We have

$$
\begin{aligned}
& \int_{\tau_{0}}^{\tau} \int_{\mathcal{O}}\left(W_{\varepsilon_{n}}(\sigma)-\alpha e^{-\sigma} \Delta W_{\varepsilon_{n}}(\sigma)\right) \wedge U_{\varepsilon_{n}}(\sigma) \cdot \operatorname{curl} \varphi(\sigma) d X d \sigma= \\
& \int_{\tau_{0}}^{\tau} \int_{\mathcal{O}}\left(W(\sigma)-\alpha e^{-\sigma} \Delta W(\sigma)\right) \wedge U(\sigma) \cdot \operatorname{curl} \varphi(\sigma) d X d \sigma+R_{n}+S_{n}
\end{aligned}
$$

where

$$
\begin{aligned}
& R_{n}=\int_{\tau_{0}}^{\tau} \int_{\mathcal{O}}\left(W_{\varepsilon_{n}}(\sigma)-\alpha e^{-\sigma} \Delta W_{\varepsilon_{n}}(\sigma)\right) \wedge\left(U(\sigma)-U_{\varepsilon_{n}}(\sigma)\right) \cdot \operatorname{curl} \varphi(\sigma) d X d \sigma, \\
& S_{n}=\int_{\tau_{0}}^{\tau} \int_{\mathcal{O}}\left(W(\sigma)-W_{\varepsilon_{n}}(\sigma)-\alpha e^{-\sigma}\left(\Delta W(\sigma)-\Delta W_{\varepsilon_{n}}(\sigma)\right)\right) \\
& \wedge U(\sigma) . \operatorname{curl} \varphi(\sigma) d X d \sigma .
\end{aligned}
$$

Due to the Hölder inequalities, the boundedness property of $W_{\varepsilon_{n}}$ in $H^{2}(\mathcal{O})^{3}$ and the inequality (2.18), we have

$$
\begin{aligned}
R_{n} & \leq C \int_{\tau_{0}}^{\tau}\left\|U(\sigma)-U_{\varepsilon_{n}}(\sigma)\right\|_{L^{\infty}(\mathcal{O})}\|\nabla \varphi(\sigma)\|_{L^{2}(\mathcal{O})} d \sigma \\
& \leq C \int_{\tau_{0}}^{\tau}\left\|W(\sigma)-W_{\varepsilon_{n}}(\sigma)\right\|_{L^{2}(\mathcal{O})}^{1 / 2}\left\|W(\sigma)-W_{\varepsilon_{n}}(\sigma)\right\|_{L^{6}(\mathcal{O})}^{1 / 2}\|\nabla \varphi(\sigma)\|_{L^{2}(\mathcal{O})} d \sigma \\
& \leq C\left(T-\tau_{0}\right) \max _{\sigma \in\left[\tau_{0}, T\right]}\left\|W(\sigma)-W_{\varepsilon_{n}}(\sigma)\right\|_{H^{1}(\mathcal{O})} \max _{\sigma \in\left[\tau_{0}, T\right]}\|\nabla \varphi(\sigma)\|_{L^{2}(\mathcal{O})} .
\end{aligned}
$$

Thus, the identity (5.10) implies that $R_{n} \rightarrow 0$ when $n \rightarrow+\infty$.

Because of the identity (5.9), it is clear that we have also $S_{n} \rightarrow 0$ when $n \rightarrow+\infty$. Thus, we have shown that, for all $\tau \in\left[\tau_{0}, \tau_{1}\right]$,

$$
\begin{aligned}
& \lim _{n \rightarrow+\infty} \int_{\tau_{0}}^{\tau} \int_{\mathcal{O}}\left(W_{\varepsilon_{n}}(\sigma)-\alpha e^{-\sigma} \Delta W_{\varepsilon_{n}}(\sigma)\right) \wedge U_{\varepsilon_{n}}(\sigma) . \operatorname{curl} \varphi(\sigma) d X d \sigma= \\
& \int_{\tau_{0}}^{\tau} \int_{\mathcal{O}}\left(W(\sigma)-\alpha e^{-\sigma} \Delta W(\sigma)\right) \wedge U(\sigma) . \operatorname{curl} \varphi(\sigma) d X d \sigma .
\end{aligned}
$$


Furthermore, since $W_{\varepsilon_{n}}(\tau)$ converge weakly to $W(\tau)$ in $\mathbb{H}^{2}(4)$, from the inequality (5.2), we get

$$
\left\|\left(I-\alpha e^{-\tau} \Delta\right)\left(W(\tau)-e^{-\tau} \sum_{i=1}^{3} b_{i} f_{i}\right)\right\|_{L^{2}(4)} \leq C \gamma^{1 / 2}\left(\frac{3}{2}-\theta\right) e^{-\theta \tau},
$$

for all $\tau \in\left[\tau_{0},+\infty\right)$.

Uniqueness. It remains to show that the solutions of (1.2) are unique in the space $C^{0}\left([0,+\infty), \mathbb{H}^{2}(4)\right)$. To show this fact, it suffices to show that the divergence free vector field $u$ obtained from a solution $w$ of (1.2) through the Biot-Savart law is unique. Since $w$ belongs to $C^{0}\left([0,+\infty), \mathbb{H}^{2}(4)\right)$, the inequality $(2.17)$ with $q=2$ and $p=\frac{6}{5}$ and the inequality (2.19) with $p=2$ of the lemma 2.4 imply directly that $u \in C^{0}\left([0,+\infty), H^{3}\left(\mathbb{R}^{3}\right)^{3}\right)$. Furthermore, $u$ satisfies the equations of motion of second grade fluids (1.1). The uniqueness of the $H^{3}$-solutions of (1.1) has been shown in [5] for the case of a bounded open set of $\mathbb{R}^{3}$ with Dirichlet boundary conditions. In our case, we can apply the computations of the proof of $[\mathbf{6}$, Theorem $2]$, which imply the uniqueness of the solutions of (1.1) with initial data in $H^{3}\left(\mathbb{R}^{3}\right)^{3}$.

\section{References}

[1] J-M. Bernard : Stationary problem of second-grade fluids in three dimensions: existence, uniqueness and regularity, Math. Methods Appl. Sci., vol 22, 1999, no. 8, p. 655-687.

[2] D. Bresch and J. Lemoine : On the existence of solutions for non-stationary second-grade fluids, Navier-Stokes equations and related nonlinear problems (Palanga, 1997), 15-30, VSP, Utrecht, 1998.

[3] A. Carpio : Asymptotic behavior for the vorticity equations in dimensions two and three, Comm. Partial Diff. Eq., vol.19, 1994, p. 827-872.

[4] A. Carpio : Large time behavior in incompressible Navier-Stokes equations, SIAM J. math. Anal., vol. 27, 1996, no. 2, p. 449-475.

[5] D. Cioranescu and V. Girault : Weak and classical solutions of a family of second grade fluids, Internat. J. Non-Linear Mech., vol. 32, 1997, no. 2, p. 317-335.

[6] D. Cioranescu and O. El Hacène : Existence and uniqueness for fluids of second grade, Nonlinear Partial Diff. Eq., Collège de France Seminar Pitman, vol. 109, 1984, p. 178-197.

[7] V. Coscia and G. Galdi : Existence and uniqueness of classical solutions of the equations of motion for second-grade fluids, Arch. Rational Mech. Anal., vol. 124, 1993, no. 3, p. 221-237.

[8] V. Coscia and G. Galdi : Existence, uniqueness and stability of regular steady motions of a second-grade fluid, Internat. J. Non-Linear Mech., vol. 29, 1994, no. 4, p. 493-506.

[9] V. Coscia and G. Galdi : Existence of regular steady motions for a second-grade fluid with non-homogeneous boundary conditions, J. Math. Sci. (New York), vol. 99, 2000, no. 1, p. 874-882.

[10] J.E. Dunn and R.L. Fosdick : Thermodynamics, stability and boundedness of fluids of complexity 2 and fluids of second grade, Arch. Rat. Mech. Anal., vol. 56, 1974, p. 191-252.

[11] M. Escobedo, O. Kavian, and H. Matano : Large time behavior of solutions of a dissipative semi-linear heat equation, Comm. Partial Differential Equations, vol. 20, 1995, p. 1427-1452.

[12] M. Escobedo and E. Zuazua : Large-time behavior for convection diffusion equations in $\mathbb{R}^{n}$, J. Funct. Anal., vol. 100, 1991, p. 119-161.

[13] V. A. Galaktionov and J. L. Vazquez: Asymptotic behaviour of nonlinear parabolic equations with critical exponents, A dynamical system approach, J. Funct. Anal., vol. 100, 1991, p. 435-462.

[14] G. Galdi, M. Grobbelaar-Van Dalsen and N. Sauer : Existence and uniqueness of classical solutions of the equations of motion for second-grade fluids, Arch. Rational Mech. Anal., vol. 124, 1993, no. 3, p. 221-237.

[15] G. Galdi, A. Sequeira and J. Videman : Steady motions of a second-grade fluid in an exterior domain, Adv. Math. Sci. Appl., vol. 7, 1997, no. 2, p. 977-995.

[16] T. Gallay and E. Wayne: Invariant Manifolds and the Long Time Asymptotics of the NavierStokes and Vorticity equations on $\mathbb{R}^{2}$, Arch. Rational Mech. Anal., vol. 163, 2002, p. 209-258. 
[17] T. Gallay and E. Wayne: Long time asymptotics of the Navier-Stokes and vorticity equations on $\mathbb{R}^{3}$, R. Soc. Lond. Philos. Trans. Ser. A Math. Phys. Eng. Sci., vol. 360, no. 1799, 2002, p. $2155-2188$.

[18] T. Gallay and E. Wayne: Global stability of vortex solutions of the two-dimensional NavierStokes equation, Comm. Math. Phys., vol. 255, 2005, no. 1, p. 97-129.

[19] T. Gallay and E. Wayne: Long time asymptotics of the Navier-Stokes equations in $\mathbb{R}^{2}$ and $\mathbb{R}^{3}$, Math. Mec., vol. 86, 2006, no. 4, p. 256-267.

[20] T. Gallay and G. Raugel : Scaling Variables and Asymptotics Expansions in Damped Wave equations, J. Diff. Eq., vol. 150, 1998, p. 42-97.

[21] T. Gallay and G. Raugel : Scaling variables and stability of hyperbolic fronts, SIAM J. Math. Anal., vol. 32, 2000, no. 1, p. 1-29.

[22] D. Henry : Geometric theory of semilinear parabolic equations, Lecture Notes in Mathematics, 1981, volume 840, Springer-Verlag edition.

[23] B. Jaffal-Mourtada : Long time asymptotics of the second grade fluid equations on $\mathbb{R}^{2}$, Dyn. of PDE, vol. 8, 2011, p. 185-223.

[24] O. Kavian : Remarks on the large time behavior of a nonlinear diffusion equation, Ann. Inst. H. Poincaré Anal. Non Linéaire, vol. 4, 1987, p. 423-452.

[25] C. Le Roux : Existence and uniqueness of the flow of second-grade fluids with slip boundary conditions, Arch. Ration. Mech. Anal., vol. 148, 1999, no. 4, p. 309-356.

[26] A. Novotný, A. Sequeira and J. Videman : Existence of three-dimensional flows of secondgrade fluids past an obstacle, Proceedings of the Second World Congress of Nonlinear Analysts, Part 5 (Athens, 1996). Nonlinear Anal., vol. 30, 1997, no. 5, p. 3051-3058.

[27] A. Pazy : Semigroups of Linear Operators and Applications to Partial Differential Equations, Appl. Math. Sci., vol. 44, Springer, 1983.

[28] R. S. Rivlin and J. L. Ericksen : Stress-deformation relations for isotropic materials, J. Rational Mech. Anal., no. 4, 1955, p. 323-425.

Department of Mathematics, Université Paris-Sud, 91405 Orsay Cedex, France E-mail address: olivier.coulaud@math.u-psud.fr 